\title{
sciendo
}

Int. J. of Applied Mechanics and Engineering, 2020, vol.25, No.3, pp.133-157

DOI: 10.2478/ijame-2020-0039

\section{NUMERICAL COMPUTATION OF LOW REYNOLDS NUMBER VISCOUS FLOW PAST BLUFF BODIES}

\author{
Md. SHAHJADA TARAFDER* and MIAD AL MURSALINE \\ Department of Naval Architecture and Marine Engineering \\ Bangladesh University of Engineering and Technology \\ E-mail: mshahjadatarafder@name.buet.ac.bd
}

\begin{abstract}
This article presents a two-dimensional steady viscous flow simulation past circular and square cylinders at low Reynolds numbers (based on the diameter) by the finite volume method with a non-orthogonal body-fitted grid. Diffusive fluxes are discretized using central differencing scheme, and for convective fluxes upwind and central differencing schemes are blended using a 'deferred correction' approach. A simplified pressure correction equation is derived, and proper under-relaxation factors are used so that computational cost is reduced without adversely affecting the convergence rate. The governing equations are expressed in Cartesian velocity components and solution is carried out using the SIMPLE algorithm for collocated arrangement of variables. The mesh yielding grid-independent solution is then utilized to study, for the very first time, the effect of the Reynolds number on the separation bubble length, separation angle, and drag coefficients for both circular and square cylinders. Finally, functional relationships between the computed quantities and Reynolds number $(\mathrm{Re})$ are proposed up to $\mathrm{Re}=40$. It is found that circular cylinder separation commences between $\mathrm{Re}=6.5-6.6$, and the bubble length, separation angle, total drag vary as $\mathrm{Re}, \mathrm{Re}^{-0.5}, \mathrm{Re}^{-0.5}$ respectively. Extrapolated results obtained from the empirical relations for the circular cylinder show an excellent agreement with established data from the literature. For a square cylinder, the bubble length and total drag are found to vary as $\operatorname{Re}$ and $\operatorname{Re}^{-0.666}$, and are greater than these for a circular cylinder at a given Reynolds number. The numerical results substantiate that a square shaped cylinder is more bluff than a circular one.
\end{abstract}

Key words: viscous flow, low Reynold's numbers, SIMPLE algorithm, circular and square cylinder, drag coefficient.

\section{Introduction}

A basic flow of great practical importance is a bluff body flow, taking place in a number of situations including moving vehicles such as cars or boats, airplanes, or flow around stationary objects such as buildings, offshore structures or cables where significant regions of a separated flow are generated. One classical bluff body problem in fluid mechanics is the flow past a circular cylinder. It has attracted the attention of many scientists and researchers owing to its application in many engineering problems such as hydrodynamic loading on ocean marine piles and offshore platform risers and support legs, and also because it can form a baseline case of more complex flows.

The cylinder flow has been investigated for more than one century, starting with the fundamental work of Strouhal [1] on the dependency between frequency of vortex shedding, free-stream velocity, and cylinder diameter. Nisi and Porter [2] carried out pioneering experimental investigation using smoke visualization and found the separation Reynolds number, $\operatorname{Re}_{s}=3.2$. Taneda [3] conducted experiments in a towing tank and investigated wakes behind the cylinder and plates photographically for the Reynolds number 0.1 to 2000. Tritton [4] revealed qualitative and quantitative features of flow at low Reynolds numbers. Grove et al. [5] performed an experimental study on fundamental characteristics of the steady separated flow past a circular cylinder paying attention to the variation of these characteristics with increasing Reynolds numbers. A series of oil tunnel experiments were carried out by Acrivos et al. [6, 7] for steady separated

\footnotetext{
* To whom correspondence should be addressed
} 
flows. Nishioka and Sato [8] determined velocity distribution across the standing eddies as well as in the wake of a cylinder at Reynolds numbers from 10 to 80. Coutanceau and Bouard [9] determined the main features of the hydrodynamic field for flow past a circular cylinder including the closed wake and the velocity distribution behind the obstacle for Reynolds numbers 5 to 40 .

The early numerical simulations past bluff bodies were carried out on steady flows employing the stream function-vorticity formulation and finite difference discretization technique. Early numerical investigations include that of Thom [10], Kawaguti [11] and Apelt [12] at $\mathrm{Re}=40$ and 44 which indicated an approximate linear growth of the standing vortex pair with the Reynolds number. Kawaguti and Jain [13] obtained the solution of nunsteady $\psi-\omega$ equation on a finite difference grid for $\operatorname{Re}=1$ to 100 . Takami and Keller [14] solved the $\psi-\omega$ equations using the finite-difference discretization method and iterative solution technique. Results were provided for drag, bubble length, shape of standing vortex, the base pressure and some formula were suggested for large Reynolds numbers. Dennis and Chang [15] used the stream function-vorticity formulation and finite difference discretization to obtain a solution for a steady incompressible flow past a cylinder up to $\mathrm{Re}=100$. Fornberg $[16,17]$ carried out a numerical investigation of a steady viscous flow around a circular cylinder employing the stream function-vorticity formulation. Henderson [18] used the spectral element method to compute viscous drag, pressure drag and base pressure for flow around a circular cylinder. Chen [19] used a penalty finite-element formulation to analyze laminar separation of flow around a cylinder bounded by two parallel plates, and investigated the effect of three types of boundary conditions. Wu et al. [20] conducted a spectral-element analysis and towing tank experiments to study the separation angle of flow around a circular cylinder at low Reynolds numbers. Sen et al. [21] carried out an extensive analysis of flow past a circular cylinder at low Reynolds numbers by stabilized a finiteelement method, employing two types of boundary conditions, namely, the no slip condition and towing tank condition. Wei et al. [22] determined the characteristics of aerodynamic forces exerted on a twisted cylinder at a low Reynolds number of 100 using finite volume discretization technique.

Another example of a classic bluff body problem is the external flow past a square cylinder which has an application in design of buildings. Okajima [23] and Okajima et al. [24] reported an extensive numerical and experimental study for an unconfined flow over a rectangular cross-section cylinder in the Reynolds number range of 100 to $2 \times 10^{4}$. The vortex shedding for a square cylinder confined in a channel was numerically studied by Mukhopadhyay et al. [25]. Sohankar et al. [26] performed calculations of an unsteady 2D flow around a square cylinder at various angle of incidence and Reynolds numbers range of 45200 using an incompressible SIMPLEC code with a non-staggered grid arrangement. Breuer et al. [27] computed a laminar flow past a square cylinder based on the lattice-Boltzmann and finite-volume method and showed that separation for a square cylinder commences at lower Reynolds numbers compared to circular cylinders. Gupta et al. [28] investigated the heat transfer and steady flow characteristics for a flow past a square cylinder by the finite difference method. Sen et al. [29] numerically computed a flow past square cylinders at low Reynolds numbers by the finite-element discretization technique and predicted the laminar separation Reynolds number for the first time to be 1.15. Mahir [30] analyzed a three-dimensional unsteady flow and heat transfer from an isothermal square cylinder subjected to cross-flow of air by using implicit fractional step solution method, third-order upwind discretization scheme for spatial derivatives and central-difference formula for viscous terms.

In the present paper, a low Reynolds number viscous flow around both a circular and square cylinder is analyzed, and empirical relations for the bubble length, drag coefficients, and separation angle are proposed by using the Finite Volume discretization and SIMPLE solution method. Despite using a simplified pressure correction equation to facilitate the solution of linear systems, a satisfactory convergence rate is achieved by a proper choice of under-relaxation factors, and considering the wide variation in results found by researchers, the present treatment predicts separated flow past bluff bodies quite accurately with low computational cost. 


\section{Mathematical modeling of fluid flow problem}

\subsection{Governing equations}

In the Cartesian co-ordinate system, the steady two-dimensional laminar flow around a cylinder for incompressible fluid is governed by the equations given by

$$
\begin{aligned}
& \frac{\partial u}{\partial x}+\frac{\partial v}{\partial y}=0 \\
& \frac{\partial(\rho u u)}{\partial x}+\frac{\partial(\rho u v)}{\partial y}=\frac{\partial}{\partial x}\left[\mu \frac{\partial u}{\partial x}\right]+\frac{\partial}{\partial y}\left[\mu \frac{\partial u}{\partial y}\right]-\frac{\partial p}{\partial x}, \\
& \frac{\partial(\rho u v)}{\partial x}+\frac{\partial(\rho v v)}{\partial y}=\frac{\partial}{\partial x}\left[\mu \frac{\partial v}{\partial x}\right]+\frac{\partial}{\partial y}\left[\mu \frac{\partial v}{\partial y}\right]-\frac{\partial p}{\partial y}
\end{aligned}
$$

where $u$ and $v$ are the velocity components in the $x$ and $y$ directions, respectively, $\rho$ is the fluid density, $P$ is the mean pressure and $\mu$ is the laminar viscosity.

Equations (2.1), (2.2), (2.3) may be represented in the following generic form

$$
\frac{\partial(\rho u \Phi)}{\partial x}+\frac{\partial(\rho v \Phi)}{\partial y}=\frac{\partial}{\partial x}\left[\Gamma \frac{\partial \Phi}{\partial x}\right]+\frac{\partial}{\partial y}\left[\Gamma \frac{\partial \Phi}{\partial y}\right]+R^{\Phi}(x, y)
$$

where $u$ and $v$ are the velocity components, $\Phi$ is any generic dependent variable $(u, v), \Gamma$ is the diffusion coefficient and $R^{\Phi}$ is the source term. Note that for the continuity equation $\Phi=1, \Gamma=0, R^{\Phi}=0$ and so on. Considering the body fitted co-ordinate system, $\xi=\xi(x, y), \eta=\eta(x, y)$ as shown in Fig.1(a and b) Eq.(2.4) can be transformed into the following form

$$
\begin{aligned}
& \frac{1}{J} \frac{\partial(\rho U \Phi)}{\partial \xi}+\frac{1}{J} \frac{\partial(\rho V \Phi)}{\partial \eta}= \\
& =\frac{1}{J} \frac{\partial}{\partial \xi}\left[\frac{\Gamma}{J}\left(\alpha \frac{\partial \Phi}{\partial \xi}-\beta \frac{\partial \Phi}{\partial \eta}\right)\right]+\frac{1}{J} \frac{\partial}{\partial \eta}\left[\frac{\Gamma}{J}\left(\gamma \frac{\partial \Phi}{\partial \eta}-\beta \frac{\partial \Phi}{\partial \xi}\right)\right]+S^{\Phi}(\xi, \eta)
\end{aligned}
$$

where

$$
U=u \frac{\partial y}{\partial \eta}-v \frac{\partial x}{\partial \eta}, \quad V=v \frac{\partial x}{\partial \xi}-u \frac{\partial y}{\partial \xi}
$$

are the contra variant velocity components.

$$
\alpha=\left(\frac{\partial x}{\partial \eta}\right)^{2}+\left(\frac{\partial y}{\partial \eta}\right)^{2}, \quad \beta=\left(\frac{\partial x}{\partial \xi}\right)\left(\frac{\partial x}{\partial \eta}\right)+\left(\frac{\partial y}{\partial \xi}\right)\left(\frac{\partial y}{\partial \eta}\right), \quad \gamma=\left(\frac{\partial x}{\partial \xi}\right)^{2}+\left(\frac{\partial y}{\partial \xi}\right)^{2}
$$

$S^{\Phi}(\xi, \eta)$ is the source term in $\xi, \eta$ coordinates, J is the Jacobian of transformation and is given by 


$$
J=\frac{\partial x}{\partial \xi} \frac{\partial y}{\partial \eta}-\frac{\partial x}{\partial \eta} \frac{\partial y}{\partial \xi}
$$

Now Eq.(2.5) is to be solved by satisfying the following boundary conditions given in Fig.2 in order to get the flow field around the cylinder.

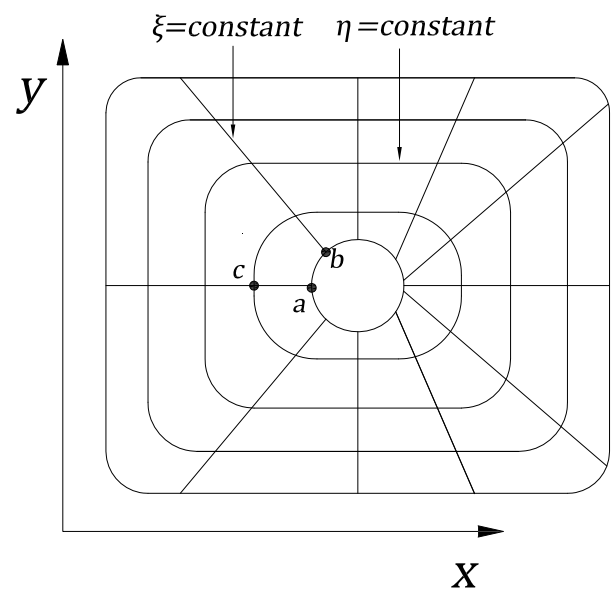

(a) Pysical domain

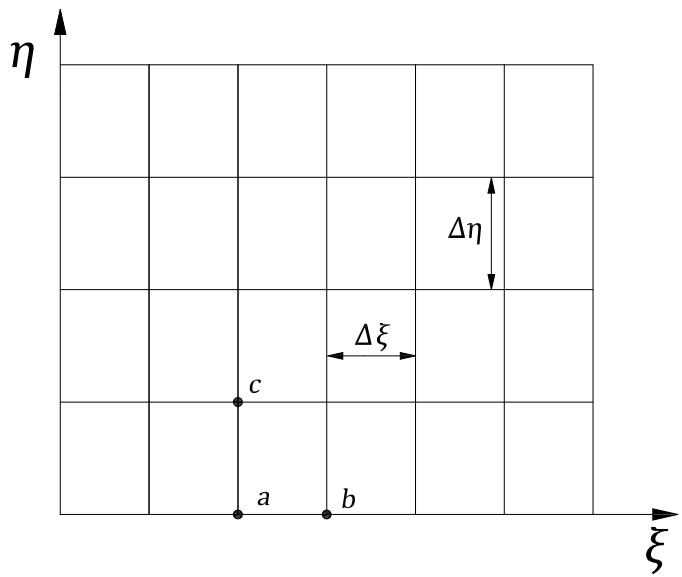

(b) Computational domain

Fig.1. Coordinate system and O-type control volume.

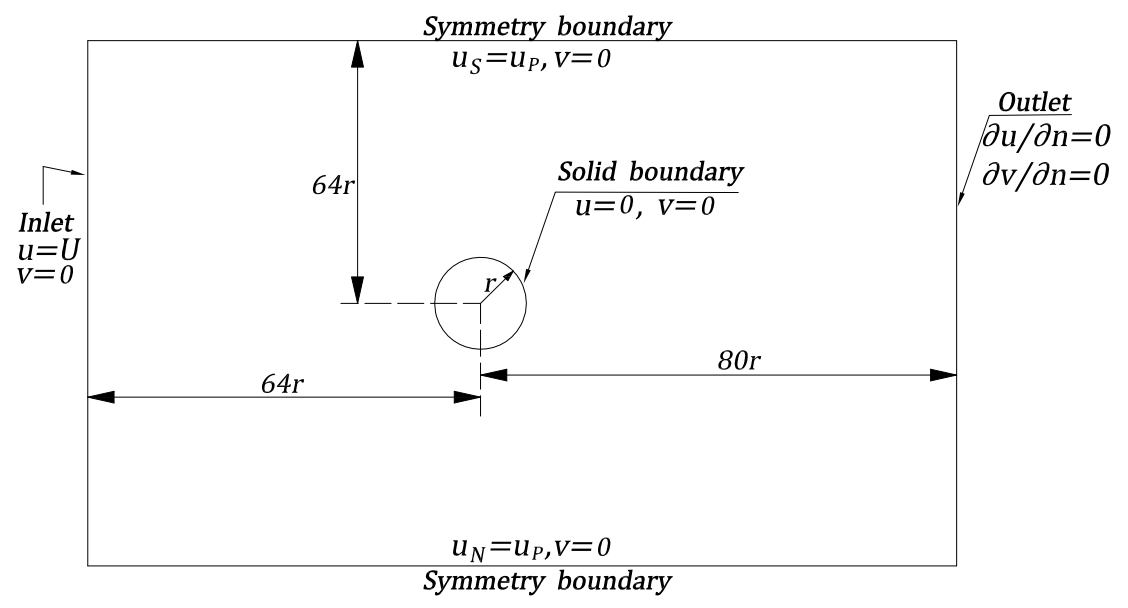

Fig.2. Problem definition and boundary conditions for circular cylinder.

\subsection{Boundary conditions}

The boundary conditions for a flow field around a cylinder fixed in a stream of uniform velocity $U$ can be written as:

(a) Inlet boundary: The components of the flow variables are provided as

$$
u=U, \quad v=0
$$


(b) Outlet boundary: The outlet boundary is located far from the region of interest and the Reynolds number is high, the gradient in the flow direction is taken to be zero. Thus

$$
\frac{\partial u}{\partial n}, \quad \frac{\partial v}{\partial n}=0
$$

where $\partial / \partial n$ is the derivative parallel to the streamlines.

(c) Solid boundary: The no slip boundary condition is applied on the surface of the body.

$$
u=0, \quad v=0 .
$$

(d) Symmetry boundary: The flow variables on the symmetry plane are prescribed as

$$
u_{S}=u_{P}, \quad u_{N}=u_{P}, \quad v=0 .
$$

\section{The finite volume method}

\subsection{Discretization of governing equation in body fitted coordinates}

The discretization is performed following a finite control volume approach in which the computational domain is divided into number contiguous quadrilateral cells. A collocated grid arrangement is used in which all the variables are stored at the geometric center of the cell. The locations of the various dependent variables and the associated cells for this grid configuration are shown in Fig.3 (a and b). Equation (2.5) is integrated over the volume of each cell in the computational domain as

$$
\begin{aligned}
& \int_{C V}\left\{\frac{\partial(\rho U \Phi)}{\partial \xi}+\frac{\partial(\rho V \Phi)}{\partial \eta}\right\} d v= \\
= & \int_{C V}\left\{\frac{\partial}{\partial \xi}\left[\frac{\Gamma}{J}\left(\alpha \Phi_{\xi}-\beta \Phi_{\eta}\right)\right]+\frac{\partial}{\partial \eta}\left[\frac{\Gamma}{J}\left(\gamma \Phi_{\eta}-\beta \Phi_{\xi}\right)\right]+J S^{\Phi}(\xi, \eta)\right\} d v .
\end{aligned}
$$

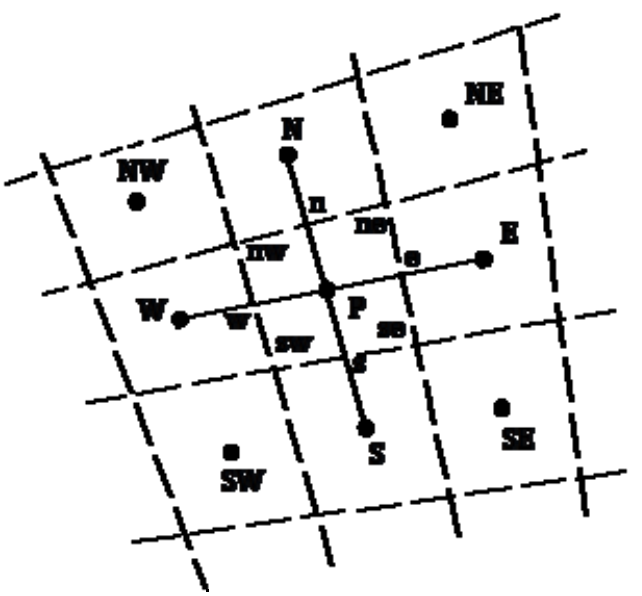

(a) Physical plane

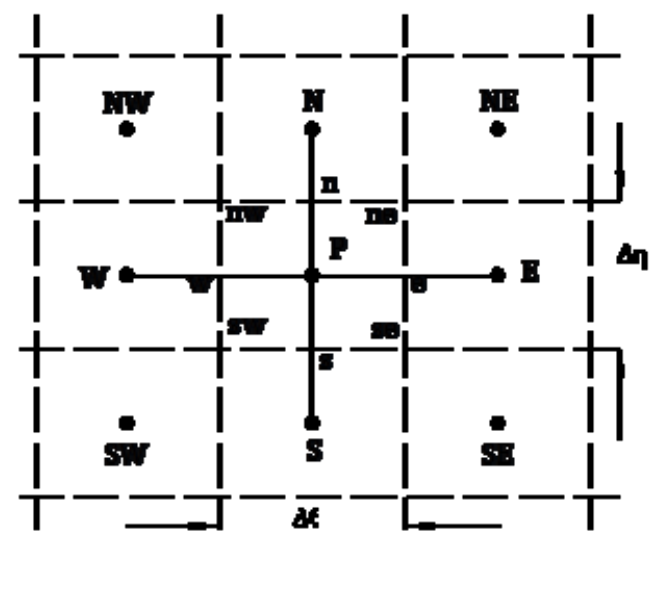

(b) Computational plane

Fig.3. Collocated grid arrangement. 
Applying Gauss's divergence theorem to convert volume integrals to surface integrals, Eq.(3.1), after little rearrangement, may be written as

$$
\begin{aligned}
& (\rho U \Phi \Delta \eta)_{w}^{e}+(\rho V \Phi \Delta \xi)_{s}^{n}=\left(\frac{\Gamma}{J} \alpha \frac{\partial \Phi}{\partial \xi} \Delta \eta\right)_{w}^{e}+\left(\frac{\Gamma}{J} \gamma \frac{\partial \Phi}{\partial \eta} \Delta \xi\right)_{s}^{n}+ \\
& +\left\{-\left(\frac{\Gamma}{J} \beta \frac{\partial \Phi}{\partial \eta} \Delta \eta\right)_{w}^{e}-\left(\frac{\Gamma}{J} \beta \frac{\partial \Phi}{\partial \xi} \Delta \xi\right)_{s}^{n}+J\left(S_{c}+S_{P} \Phi_{P}\right) \Delta \xi \Delta \eta\right\} .
\end{aligned}
$$

The cross derivative terms have been added to the source term which in turn has been linearized as suggested by Patanker [31]. Using the notation of Fig.3, the following approximations may be made for the derivatives at face $e$

$$
\begin{aligned}
& \left(\frac{\partial x}{\partial \xi}\right)_{e} \approx \frac{x_{E}-x_{P}}{\xi_{E}-\xi_{P}},\left(\frac{\partial x}{\partial \eta}\right)_{e} \approx \frac{\left(x_{n}-x_{s}\right)_{e}}{\left(\eta_{n}-\eta_{s}\right)_{e}}=\frac{x_{n e}-x_{s e}}{\eta_{n e}-\eta_{s e}} \\
& \left(\frac{\partial y}{\partial \xi}\right)_{e} \approx \frac{y_{E}-y_{P}}{\xi_{E}-\xi_{P}},\left(\frac{\partial y}{\partial \eta}\right)_{e} \approx \frac{\left(y_{n}-y_{s}\right)_{e}}{\left(\eta_{n}-\eta_{s}\right)_{e}}=\frac{y_{n e}-y_{s e}}{\eta_{n e}-\eta_{s e}} .
\end{aligned}
$$

Analogous expressions may be derived for other faces. Using Eqs (2.6), (2.7), (2.8) and Eq.(3.3), Eq.(3.2) can be written as

$$
\begin{aligned}
& {\left[F_{e} \Phi_{e}-F_{w} \Phi_{w}+F_{n} \Phi_{n}-F_{s} \Phi_{s}\right]=} \\
& =D_{e}\left(\Phi_{E}-\Phi_{P}\right)-D_{w}\left(\Phi_{P}-\Phi_{W}\right)+D_{n}\left(\Phi_{N}-\Phi_{P}\right)-D_{s}\left(\Phi_{P}-\Phi_{s}\right)+ \\
& +\left[N_{e}\left(\Phi_{n}-\Phi_{s}\right)_{e}-N_{w}\left(\Phi_{n}-\Phi_{s}\right)_{w}+N_{n}\left(\Phi_{e}-\Phi_{w}\right)_{n}-N_{s}\left(\Phi_{e}-\Phi_{w}\right)_{s}+\left(S_{c}+S_{P} \Phi_{P}\right) \delta V\right]
\end{aligned}
$$

Central differencing is used to discretize the diffusion terms and a suitable interpolation for the convective terms is required to express cell face values in terms of nodal values. This is achieved as in Demirdzic et al. [32] by blending second-order central (CDS) differencing and first-order unconditionally stable upwind differencing scheme (UDS) in a deferred correction manner

$$
\left[\Phi_{e}^{U D S}\right]_{\text {implicit }}+\lambda\left[\Phi_{e}^{C D S}-\Phi_{e}^{U D S}\right]_{\text {explicit }}
$$

where $\lambda$ is the blending factor having value between 0 to 1 . The explicit part in Eq.(3.5) is obtained from previous iteration and added to the source term, like the cross derivative terms. Using the above scheme for convective terms and after little manipulation, Eq.(3.4) can be written in the following algebraic form

$$
a_{P} \Phi_{P}=a_{W} \Phi_{W}+a_{E} \Phi_{E}+a_{N} \Phi_{N}+a_{S} \Phi_{S}+S^{\prime}
$$

Introducing an under-relaxation factor to slow down changes of a dependent variable in consecutive iterations, Eq.(3.6) becomes 


$$
\begin{aligned}
& \left(\frac{a_{P}}{\alpha_{\Phi}}\right) \Phi_{P}=a_{W} \Phi_{W}+a_{E} \Phi_{E}+a_{N} \Phi_{N}+a_{S} \Phi_{S}+S_{\Phi} \\
& \left(\frac{a_{P}}{\alpha_{\Phi}}\right) \Phi_{P}=\sum_{n b} a_{n b} \Phi_{n b}+S_{\Phi}
\end{aligned}
$$

where

$$
\begin{aligned}
& \sum_{n b} a_{n b} \Phi_{n b}=a_{W} \Phi_{W}+a_{E} \Phi_{E}+a_{N} \Phi_{N}+a_{S} \Phi_{S}, \\
& a_{P}=a_{W}+a_{E}+a_{N}+a_{S}-\left(S_{P}\right) \delta V+\left[F_{e}-F_{w}+F_{n}-F_{S}\right]=a_{E}+a_{N}+a_{S}-\left(S_{P}\right) \delta V .
\end{aligned}
$$

The term in brackets corresponds to the continuity equation. After outer iteration steps, the mass fluxes are corrected so that the bracketed term vanishes identically and, therefore, are not considered.

$$
\begin{aligned}
& S_{\Phi}=S^{\prime}+\frac{\left(1-\alpha_{\Phi}\right)}{\alpha_{\Phi}} a_{P} \Phi_{P}^{m}= \\
& =N_{e}\left(\Phi_{n}-\Phi_{s}\right)_{e}-N_{w}\left(\Phi_{n}-\Phi_{s}\right)_{w}+N_{n}\left(\Phi_{e}-\Phi_{w}\right)_{n}-N_{s}\left(\Phi_{e}-\Phi_{w}\right)_{s}+ \\
& +\lambda\left\{\left[F_{e} \Phi_{e}-F_{w} \Phi_{w}+F_{n} \Phi_{n}-F_{s} \Phi_{s}\right]_{U D S}-\left[F_{e} \Phi_{e}-F_{w} \Phi_{w}+F_{n} \Phi_{n}-F_{s} \Phi_{s}\right]_{C D S}\right\}+ \\
& +\left(S_{c}\right) \delta V+\text { pterm }+\frac{\left(1-\alpha_{\Phi}\right)}{\alpha_{\Phi}} a_{P} \Phi_{P}^{m}, \\
& \text { pterm }\left\{\begin{array}{c}
=-\left(p_{e}-p_{w}\right)\left(y_{n}-y_{s}\right)+\left(p_{n}-p_{s}\right)\left(y_{e}-y_{w}\right) \text { for } x-\text { momentum } \\
=-\left(p_{n}-p_{s}\right)\left(x_{e}-x_{w}\right)+\left(p_{e}-p_{w}\right)\left(x_{n}-x_{s}\right) \text { for } y \text { - momentum } \\
=0 \text { for continuity equation }
\end{array}\right.
\end{aligned}
$$

\begin{tabular}{|c|c|c|c|}
\hline$a_{W}$ & $a_{E}$ & $a_{N}$ & $a_{S}$ \\
\hline$D_{w}+\max \left(F_{w}, 0\right)$ & $D_{e}+\max \left(0,-F_{e}\right)$ & $D_{n}+\max \left(0,-F_{n}\right)$ & $D_{s}+\max \left(F_{s}, 0\right)$ \\
\hline
\end{tabular}

$$
\begin{aligned}
& D_{e}=\frac{\Gamma\left\{\left(x_{n}-x_{s}\right)_{e}^{2}+\left(y_{n}-y_{s}\right)_{e}^{2}\right\}}{\left(x_{E}-x_{P}\right)\left(y_{n}-y_{s}\right)_{e}-\left(x_{n}-x_{s}\right)_{e}\left(y_{E}-y_{P}\right)}, \\
& D_{w}=\frac{\Gamma\left\{\left(x_{n}-x_{s}\right)_{w}^{2}+\left(y_{n}-y_{s}\right)_{w}^{2}\right\}}{\left(x_{P}-x_{W}\right)\left(y_{n}-y_{s}\right)_{w}-\left(x_{n}-x_{s}\right)_{w}\left(y_{P}-y_{W}\right)}, \\
& D_{n}=\frac{\Gamma\left\{\left(x_{e}-x_{w}\right)_{n}^{2}+\left(y_{e}-y_{w}\right)_{n}^{2}\right\}}{-\left(x_{N}-x_{P}\right)\left(y_{e}-y_{w}\right)_{n}+\left(x_{e}-x_{w}\right)_{n}\left(y_{N}-y_{P}\right)}, \\
& D_{s}=\frac{\Gamma\left\{\left(x_{e}-x_{w}\right)_{s}^{2}+\left(y_{e}-y_{w}\right)_{s}^{2}\right\}}{-\left(x_{P}-x_{S}\right)\left(y_{e}-y_{w}\right)_{s}+\left(x_{e}-x_{w}\right)_{s}\left(y_{P}-y_{S}\right)},
\end{aligned}
$$




$$
\begin{aligned}
& F_{e}=\rho\left\{u_{e}\left(y_{n}-y_{s}\right)_{e}-v_{e}\left(x_{n}-x_{s}\right)_{e}\right\}, F_{w}=\rho\left\{u_{w}\left(y_{n}-y_{s}\right)_{w}-v_{w}\left(x_{n}-x_{s}\right)_{w}\right\}, \\
& F_{n}=\rho\left\{-u_{n}\left(y_{e}-y_{w}\right)_{n}+v_{n}\left(x_{e}-x_{w}\right)_{n}\right\}, F_{s}=\rho\left\{-u_{s}\left(y_{e}-y_{w}\right)_{s}+v_{s}\left(x_{e}-x_{w}\right)_{s}\right\}, \\
& N_{e}=\frac{\Gamma\left\{\left(x_{E}-x_{P}\right)\left(x_{n}-x_{s}\right)_{e}+\left(y_{E}-y_{P}\right)\left(y_{n}-y_{s}\right)_{e}\right\}}{\left(x_{n}-x_{s}\right)_{e}\left(y_{E}-y_{P}\right)-\left(x_{E}-x_{P}\right)\left(y_{n}-y_{s}\right)_{e}}, \\
& N_{w}=\frac{\Gamma\left\{\left(x_{P}-x_{W}\right)\left(x_{n}-x_{s}\right)_{w}+\left(y_{P}-y_{W}\right)\left(y_{n}-y_{s}\right)_{w}\right\}}{\left(x_{n}-x_{s}\right)_{w}\left(y_{P}-y_{W}\right)-\left(x_{P}-x_{W}\right)\left(y_{n}-y_{s}\right)_{w}}, \\
& N_{n}=\frac{\Gamma\left\{\left(x_{N}-x_{P}\right)\left(x_{e}-x_{w}\right)_{n}+\left(y_{N}-y_{P}\right)\left(y_{e}-y_{w}\right)_{n}\right\}}{\left(x_{N}-x_{P}\right)\left(y_{e}-y_{w}\right)_{n}-\left(x_{e}-x_{w}\right)_{n}\left(y_{N}-y_{P}\right)}, \\
& N_{s}=\frac{\Gamma\left\{\left(x_{P}-x_{S}\right)\left(x_{e}-x_{w}\right)_{s}+\left(y_{P}-y_{S}\right)\left(y_{e}-y_{w}\right)_{s}\right\}}{\left(x_{P}-x_{S}\right)\left(y_{e}-y_{w}\right)_{s}-\left(x_{e}-x_{w}\right)_{s}\left(y_{P}-y_{S}\right)},
\end{aligned}
$$

and the volume (area in 2D) of the cell $\delta V$ around the node $\mathrm{P}$ as indicated in Fig.3a is

$$
\begin{aligned}
& \delta V=\left[\left(x_{e}-x_{w}\right)\left(y_{n}-y_{s}\right)-\left(x_{n}-x_{s}\right)\left(y_{e}-y_{w}\right)\right]= \\
& =\frac{1}{2}\left[\left(x_{n e}-x_{s w}\right)\left(y_{n w}-y_{s e}\right)-\left(y_{n e}-y_{s w}\right)\left(x_{n w}-x_{s e}\right)\right] .
\end{aligned}
$$

$\Phi_{P}^{m}$ denotes value of dependent variable from previous iteration and $\alpha_{\Phi}$ is the under relaxation factor.

\section{Solution by SIMPLE algorithm}

To obtain velocity and pressure fields, an iterative solution procedure akin to the SIMPLE method by Patankar and Spalding [33] is used. In the present work, the scalar and vector variables are stored in a collocated manner. Using Eq.(3.8), the momentum equations in the $x$ and $y$ directions may be written as

$$
\begin{aligned}
& \left(\frac{a_{P}}{\alpha_{u}}\right) u_{P}=\sum a_{n b} u_{n b}+S_{u}-\left(p_{e}-p_{w}\right)\left(y_{n}-y_{s}\right)+\left(p_{n}-p_{s}\right)\left(y_{e}-y_{w}\right), \\
& \left(\frac{a_{P}}{\alpha_{v}}\right) v_{P}=\sum a_{n b} u_{n b}+S_{v}+\left(p_{e}-p_{w}\right)\left(x_{n}-x_{s}\right)-\left(p_{n}-p_{s}\right)\left(x_{e}-x_{w}\right) .
\end{aligned}
$$

The pressure terms have been removed from the source terms in the above equations for convenience. Equations (4.1) and (4.2) may be written in the following matrix form.

$$
\left[\boldsymbol{A}_{u}\right]\{\boldsymbol{u}\}=\left\{\boldsymbol{S}_{\boldsymbol{u}}\right\}
$$




$$
\left[\boldsymbol{A}_{\boldsymbol{v}}\right]\{\boldsymbol{v}\}=\left\{\boldsymbol{S}_{\boldsymbol{v}}\right\}
$$

where $\{\boldsymbol{u}\},\{\boldsymbol{v}\}$ denotes the field of the unknown nodal velocity field arranged in a vector form, $\{\boldsymbol{S}\}$ is a similar column vector containing source terms and $[\boldsymbol{A}]$ is the coefficient matrix. For the $(m+1)^{\text {th }}$ outer iteration, the matrix $\left[\boldsymbol{A}_{\boldsymbol{u}}\right]$ and column vector $\boldsymbol{S}_{\boldsymbol{u}}$ are obtained using the tentative values of the parameters $u^{m}, v^{m}, P^{m}$. These parameters superscripted by $m$ are either initial guesses or solution to the governing equations at the $m^{\text {th }}$ outer iteration. Once matrix $\left[\boldsymbol{A}_{\boldsymbol{u}}\right]$ and column vector $\boldsymbol{S}_{\boldsymbol{u}}$ are assembled, the system of equations represented by Eq.(4.3) is solved within the inner iteration loop by the strongly implicit procedure of Stone [34]. The velocity field $u^{*}$, obtained in this manner satisfies the following equation

$$
\left[A_{u}\right]\left\{u^{*}\right\}=\left\{S_{u}\right\}
$$

The asterisk is used to indicate that the computed velocity field satisfies momentum but not necessarily the continuity equation. Similarly $v^{*}$ satisfies the following system

$$
\left[A_{u}\right]\left\{v^{*}\right\}=\left\{S_{v}\right\}
$$

However, the success of the SIMPLE algorithm in the case of collocated arrangement of variables depends on the interpolation of nodal velocities to obtain face velocities and hence mass fluxes. To avoid false pressure field a special interpolation technique suggested by Rhie [35] is employed which leads to the following $u$ velocity at face $e$

$$
u_{e}^{*}=\alpha_{u}\left[\overline{\frac{\sum a_{n b} u_{n b}^{*}+B_{P}}{a_{P}}}\right]_{e}-\alpha_{u} \overline{\left(\frac{1}{a_{P}}\right)_{e}}\left(y_{n}-y_{s}\right)_{e}\left(P_{E}^{m}-P_{P}^{m}\right)
$$

where $B_{P}$ is the source term excluding pressure gradient across the cell. The over bar denotes linear interpolation between two neighboring nodes which for an arbitrary quantity $g$ is given by

$$
g=f_{e}^{+} g+\left(1-f_{e}^{+}\right) g
$$

where

$$
f_{e}^{+}=\frac{|\overline{P e}|}{|\overline{P e}|+|\overline{e E}|}
$$

Similarly, velocities at other faces may be obtained in both $x$ and $y$ directions. The mass fluxes obtained from these face velocities are not guaranteed to satisfy the discrete continuity equation. That is

$$
\begin{aligned}
& F_{e}^{*}-{F_{w}}^{*}+{F_{n}}^{*}-F_{s}^{*} \neq 0, \\
& {F_{e}}^{*}-{F_{w}}^{*}+{F_{n}}^{*}-{F_{s}}^{*}=S_{m} .
\end{aligned}
$$

Consequently, the face velocities are corrected in the spirit of the SIMPLE algorithm as follows 


$$
\begin{aligned}
& u_{e} \approx u_{e}^{*}+u_{e}^{\prime} \text {, } \\
& u_{e} \approx u_{e}^{*}+\alpha_{u}\left(y_{e}-y_{w}\right)_{e} \frac{\overline{\left(p_{n}^{\prime}-p_{s}^{\prime}\right)_{e}}}{\left(a_{P}\right)_{e}}-\alpha_{u}\left(y_{n}-y_{s}\right)_{e} \overline{\left(\frac{1}{a_{P}}\right)_{e}}\left(p_{E}^{\prime}-p_{P}^{\prime}\right), \\
& v_{e} \approx v_{e}^{*}+v_{e}^{\prime}, \\
& v_{e} \approx v_{e}^{*}-\alpha_{v}\left(x_{e}-x_{w}\right)_{e} \overline{\overline{\left(p_{n}^{\prime}-p_{s}^{\prime}\right)_{e}}}+\alpha_{v}\left(x_{n}-x_{s}\right)_{e} \overline{\left(\frac{1}{\left.a_{P}\right)_{e}}\right)_{e}}\left(p_{E}^{\prime}-p_{P}^{\prime}\right) .
\end{aligned}
$$

Using Eqs (4.11) and (4.12) the mass flux at face $e$ is found to be

$$
\begin{aligned}
& F_{e}=\rho\left\{-\alpha_{u} \overline{\left(\frac{1}{a_{P}}\right)_{e}}\left(y_{n}-y_{s}\right)_{e}^{2}\left(p_{E}^{\prime}-p_{P}^{\prime}\right)-\alpha_{v} \overline{\left(\frac{1}{a_{P}}\right)_{e}}\left(x_{n}-x_{s}\right)_{e}^{2}\left(p_{E}^{\prime}-p_{P}^{\prime}\right)\right\}+ \\
& +\rho\left\{\alpha_{u}\left(y_{e}-y_{w}\right)_{e}\left(y_{n}-y_{s}\right)_{e} \frac{\overline{\left(p_{n}^{\prime}-p_{s}^{\prime}\right)_{e}}}{\left(a_{P}\right)_{e}}+\alpha_{v}\left(x_{n}-x_{s}\right)_{e}\left(x_{e}-x_{w}\right)_{e} \frac{\overline{\left(p_{n}^{\prime}-p_{s}^{\prime}\right)_{e}}}{\left(a_{P}\right)_{e}}\right\}+ \\
& +\rho\left[u^{*}{ }_{e}\left(y_{n}-y_{s}\right)_{e}-v_{e}^{*}\left(x_{n}-x_{s}\right)_{e}\right] .
\end{aligned}
$$

Neglecting the second term (cross-derivative contribution) of Eq.(4.13) based on the recommendation of Peric [36] we get

$$
\begin{aligned}
& F_{e}=-\rho\left\{\alpha_{u} \overline{\left(\frac{1}{a_{P}}\right)_{e}}\left(y_{n}-y_{s}\right)_{e}^{2}+\alpha_{v} \overline{\left(\frac{1}{a_{P}}\right)_{e}}\left(x_{n}-x_{s}\right)_{e}^{2}\right\}\left(p_{E}^{\prime}-p_{P}^{\prime}\right)+ \\
& +\rho\left[u^{*}{ }_{e}\left(y_{n}-y_{s}\right)_{e}-v^{*}{ }_{e}\left(x_{n}-x_{s}\right)_{e}\right] .
\end{aligned}
$$

Similarly, we can write for other faces

$$
\begin{aligned}
& F_{w}=-\rho\left\{\alpha_{u} \overline{\left(\frac{1}{a_{P}}\right)_{w}}\left(y_{n}-y_{s}\right)_{w}^{2}+\alpha_{v} \overline{\left(\frac{1}{a_{P}}\right)_{w}}\left(x_{n}-x_{s}\right)_{w}^{2}\right\}\left(p_{P}^{\prime}-p_{W}^{\prime}\right)+ \\
& +\rho\left[u_{w}^{*}\left(y_{n}-y_{s}\right)_{w}-v_{w}^{*}\left(x_{n}-x_{s}\right)_{w}\right], \\
& F_{n}=-\rho\left\{\alpha_{u} \overline{\left(\frac{1}{a_{P}}\right)_{n}}\left(y_{e}-y_{w}\right)_{n}^{2}+\alpha_{v} \overline{\left(\frac{1}{a_{P}}\right)_{n}}\left(x_{e}-x_{w}\right)_{n}^{2}\right\}\left(p_{N}^{\prime}-p_{P}^{\prime}\right)+ \\
& +\rho\left[-u_{n}^{*}\left(y_{e}-y_{w}\right)_{n}+v_{n}^{*}\left(x_{e}-x_{w}\right)_{n}\right], \\
& F_{S}=-\rho\left\{\alpha_{u} \overline{\left(\frac{1}{a_{P}}\right)_{S}}\left(y_{e}-y_{w}\right)_{s}^{2}+\alpha_{v} \overline{\left(\frac{1}{a_{P}}\right)_{S}}\left(x_{e}-x_{w}\right)_{s}^{2}\right\}\left(p_{P}^{\prime}-p_{S}^{\prime}\right)+ \\
& +\rho\left[-u_{s}^{*}\left(y_{e}-y_{w}\right)_{s}+v_{s}^{*}\left(x_{e}-x_{w}\right)_{s}\right] .
\end{aligned}
$$


Substituting Eq.(4.14) to Eq.(4.17) in the discretized continuity Eq.(4.18) for an incompressible flow, the simplified pressure correction Eq.(4.19) without cross-derivative contributions is derived after algebraic manipulation.

where

$$
\begin{aligned}
& F_{e}-F_{w}+F_{n}-F_{s}=0, \\
& a_{P} p_{P}^{\prime}=a_{W} p_{W}^{\prime}+a_{E} p_{E}^{\prime}+a_{N} p_{S}^{\prime}+a_{S} p_{N}^{\prime}+S_{m}
\end{aligned}
$$

$$
\begin{aligned}
& a_{P}=a_{W}+a_{E}+a_{N}+a_{S}, \\
& a_{E}=\rho\left\{\alpha_{u} \overline{\left(\frac{1}{a_{P}}\right)_{e}}\left(y_{n}-y_{s}\right)_{e}^{2}+\alpha_{v} \overline{\left(\frac{1}{a_{P}}\right)_{e}}\left(x_{n}-x_{s}\right)_{e}^{2}\right\}, \\
& a_{W}=\rho\left\{\alpha_{u} \overline{\left(\frac{1}{a_{P}}\right)_{w}}\left(y_{n}-y_{s}\right)_{w}^{2}+\alpha_{v} \overline{\left(\frac{1}{a_{P}}\right)_{w}}\left(x_{n}-x_{s}\right)_{w}^{2}\right\}, \\
& a_{N}=\rho\left\{\alpha_{u} \overline{\left(\frac{1}{a_{P}}\right)_{n}}\left(y_{e}-y_{w}\right)_{n}^{2}+\alpha_{v}\left(\frac{1}{a_{P}}\right)_{n}\left(x_{e}-x_{w}\right)_{n}^{2}\right\}, \\
& a_{S}=\rho\left\{\alpha_{u} \overline{\left(\frac{1}{a_{P}}\right)_{s}}\left(y_{e}-y_{w}\right)_{s}^{2}+\alpha_{v}\left(\frac{1}{a_{P}}\right)_{s}\left(x_{e}-x_{w}\right)_{s}^{2}\right\}, \\
& S_{m}=\left[\left[u_{w}^{*}\left(y_{n}-y_{s}\right)_{w}-v_{w}^{*}\left(x_{n}-x_{s}\right)_{w}\right]\right]_{-}\left[u_{e}^{*}\left(y_{n}-y_{s}\right)_{e}-v_{e}^{*}\left(x_{n}-x_{s}\right)_{e}\right]+ \\
& +\left[-u_{s}^{*}\left(y_{e}-y_{w}\right)_{s}+v_{s}^{*}\left(x_{e}-x_{w}\right)_{s}\right]-\left[-u_{n}^{*}\left(y_{e}-y_{w}\right)_{n}+v_{n}^{*}\left(x_{e}-x_{w}\right)_{n}\right] .
\end{aligned}
$$

Pressure corrections are obtained by solving Eq.(4.19) by the ILU decomposition method of Stone [34]. These pressure corrections may be in turn used to correct mass fluxes, nodal velocities and pressure as follows

$$
\begin{aligned}
& F_{e}^{m+1}=u_{e}^{*}\left(y_{n}-y_{s}\right)_{e}-v_{e}^{*}\left(x_{n}-x_{s}\right)_{e}+ \\
& -\rho\left\{\alpha_{u} \overline{\left(\frac{1}{a_{P}}\right)_{e}}\left(y_{n}-y_{s}\right)_{e}^{2}+\alpha_{v} \overline{\left(\frac{1}{a_{P}}\right)_{e}}\left(x_{n}-x_{s}\right)_{e}^{2}\right\}\left(p_{E}^{\prime}-p_{P}^{\prime}\right), \\
& u_{P}^{m+1}=u_{P}^{*}-\alpha_{u}\left(y_{n}-y_{s}\right)_{e} \overline{\left(\frac{1}{a_{P}}\right)_{e}}\left(p_{E}^{\prime}-p_{P}^{\prime}\right), \\
& p^{m+1}=p^{m}+\alpha_{p} p^{\prime} .
\end{aligned}
$$

This marks the completion of the $(m+1)^{t h}$ outer iteration and the obtained flow variables $u^{m+1}, v^{m+1}, \mathrm{p}^{m+1}$ act as 'initial guesses' for the $(m+2)^{t h}$ outer iteration and the whole process described above is repeated until the convergence criterion is met. 


\section{Convergence criteria}

Starting from the initial guess for all field values the process of solving the equations is repeated until convergence. Due to coupling of variables and the nonlinearity of the equations, it is not necessary to solve exactly the discretized equations for a given set of coefficients (inner iteration); these are only approximate and need to be updated. So, inner iterations of momentum equations are terminated by limiting the number of iteration to 1 . Convergence of the pressure correction equation is monitored by comparing the sum of the absolute residuals after each sweep to its initial value.

For outer iterations (solution with updated coefficients), the sum of the absolute values of the residuals over all control volumes is calculated and normalized by the inlet flux of the relevant quantity, $f_{\varphi}^{\text {inlet }}$, that is

$$
\bar{R}_{\varphi}=\frac{\sum_{l=1}^{K} R_{\varphi}^{l}}{f_{\varphi}^{\text {inlet }}} .
$$

For convergence of outer iteration to take place the following must be satisfied

$$
\max \left(\bar{R}_{u}, \bar{R}_{v}, \bar{R}_{l}\right) \leq \lambda .
$$

The above criterion ensures that the relative changes in the variables from one iteration to the next are of the order of $\lambda$ or less.

\section{Results and discussion}

\subsection{Flow past a circular cylinder}

Viscous flow past a single cylinder with unit diameter and zero incidence is simulated at low Reynolds numbers $(\mathrm{Re})$ with O-type grids of sizes $48 \times 32,96 \times 64$, and $192 \times 128$. The computational domain details are shown in Fig.2, and Fig.4 shows the finest grid. The measurement of Taneda [3], Coutanceau and Bouard [9] and Homann [37] show that the flow separation on the cylinder surface commences at the Reynolds number $\left(\operatorname{Re}_{s}\right) 5$ to 7 depending on the blockage ratio $\mathrm{B}$ (ratio of the cylinder diameter and the domain width). It is evident from Fig.6a that the onset of separation is between $\mathrm{Re}=6.5-6.6$ for the present case with $B=0.016$. The increase in length of the bubble with the Reynolds number can be observed from Fig.6b. Pressure coefficients are calculated for Reynolds numbers of 15, 30, 40, 100, as shown in Fig.7, which agree well with results from the literature. With the $192 \times 128$ grid, the surface pressure coefficients tend to show grid independency, and a further refinement of the grid is thus considered redundant. The influence of the Reynolds number on the surface pressure coefficient for the cylinder is illustrated in Fig.8 using the finest mesh. The separation angle, $\theta_{S}$ (defined in Fig.5), is computed for various Reynolds numbers and compared with Coutanceau and Bouard [9] and Takami and Keller [14]. The results agree well with Takami and Keller [14] at all Reynolds number but disagree slightly with Coutanceau and Bouard [9] at Re $=10$ (see Fig.9). The correlation of the bubble length and Reynolds number is found to be positive and linear (Fig.10) as reported by earlier researchers. The bubble length in the present numerical computation is found to be slightly lower than in Taneda [3] and slightly higher than in Coutanceau and Bouard [9]. The variation of coefficients of viscous drag, pressure drag and total drag with the Reynolds number is illustrated in Fig.11. The discrepancy in drag coefficients is not very significant and the values are only slightly overestimated. 

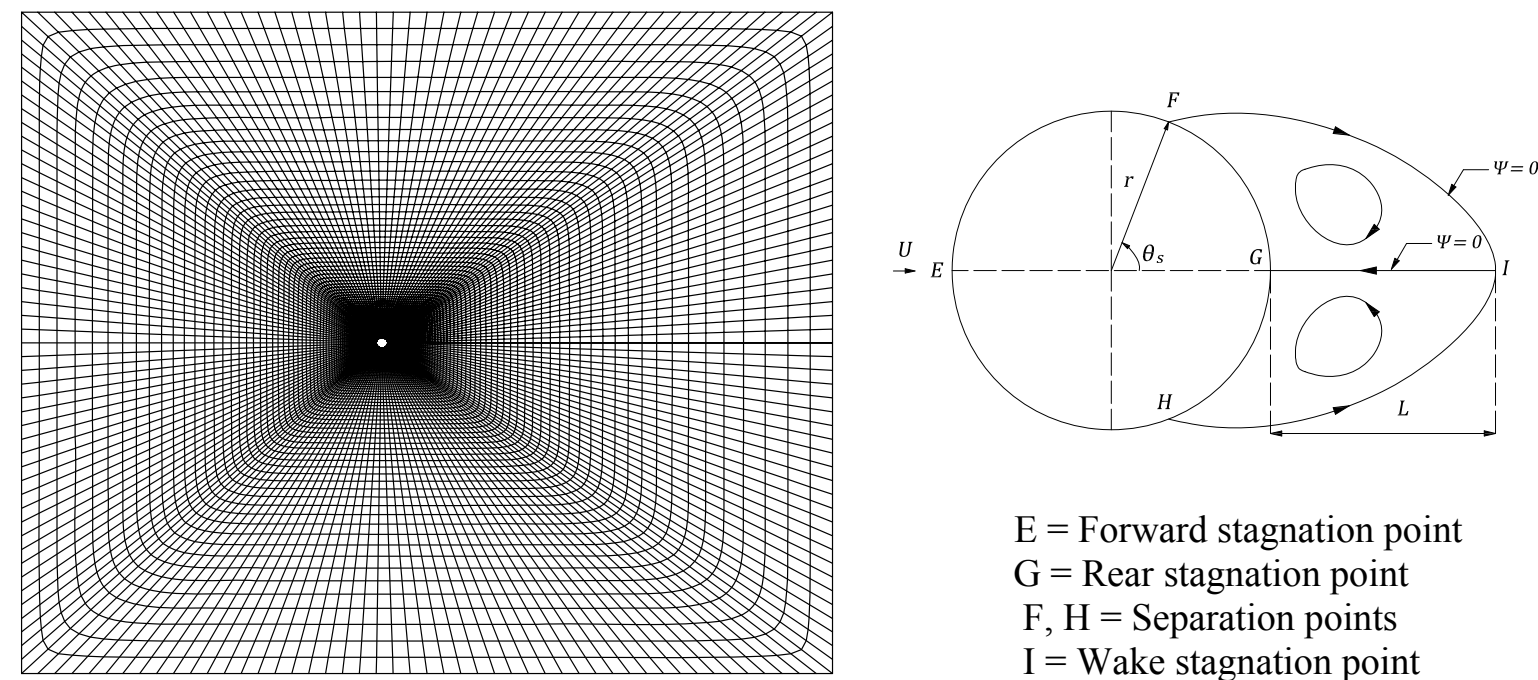

$\mathrm{E}=$ Forward stagnation point

$\mathrm{G}=$ Rear stagnation point

$\mathrm{F}, \mathrm{H}=$ Separation points

$\mathrm{I}=$ Wake stagnation point

Fig.4. Overview of O-type grid used for a circular Fig.5. Schematic representation of the separation cylinder.

bubble cross-section for circular cylinder.

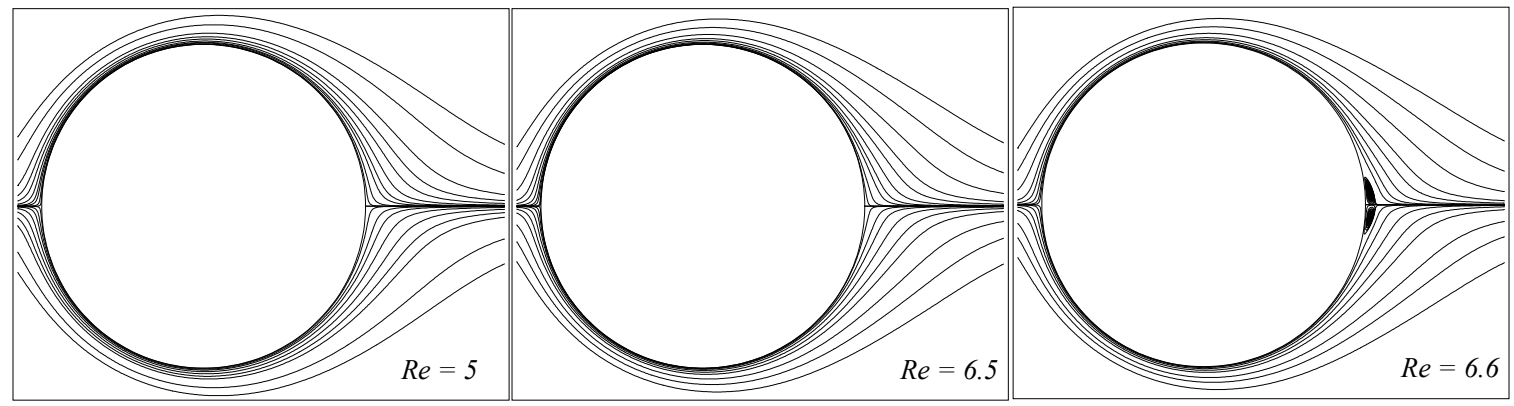

(a)

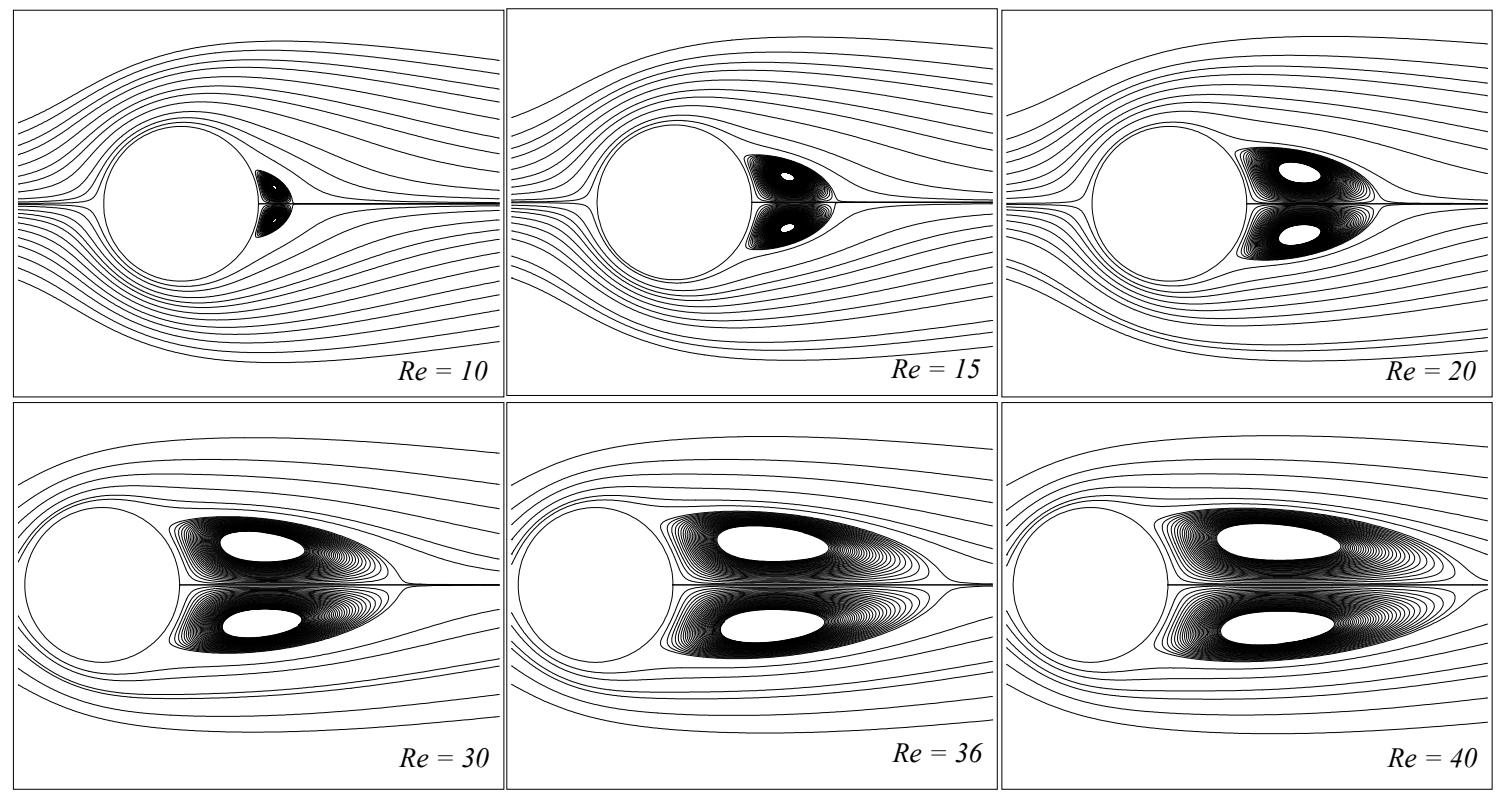

Fig.6. (a) Onset of separation (b) Change of separation bubble with Reynolds number. 


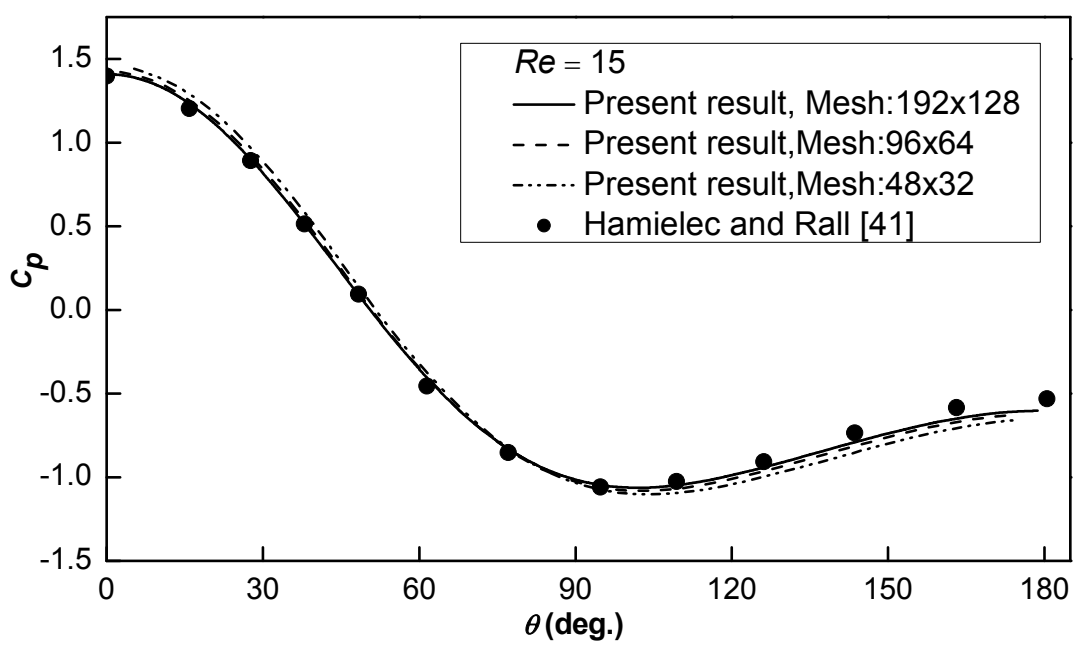

Fig.7a. Pressure coefficients for circular cylinder at (a) $\operatorname{Re}=15$.

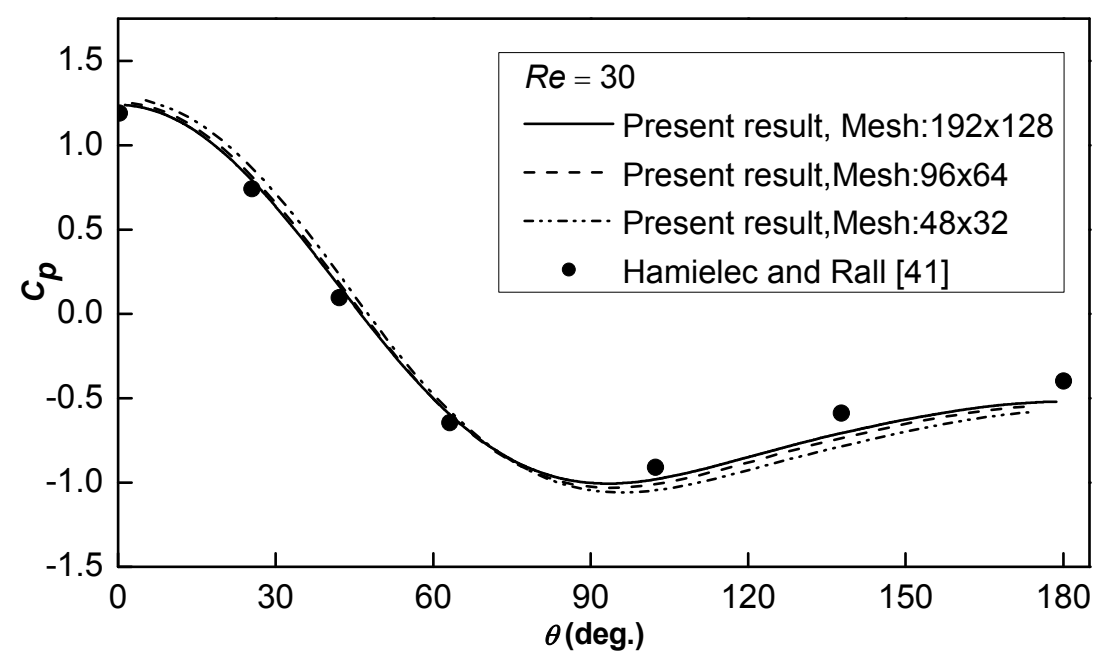

Fig.7b. Pressure coefficients for circular cylinder at (b) $\mathrm{Re}=30$.

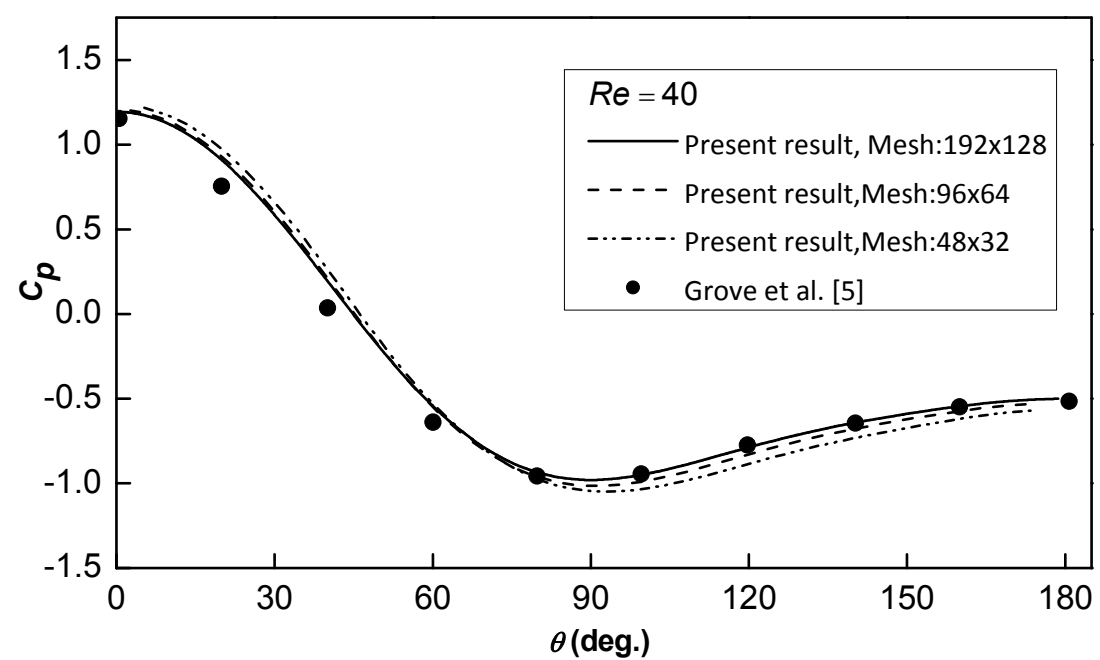

Fig.7c. Pressure coefficients for circular cylinder at (c) $\operatorname{Re}=40$. 


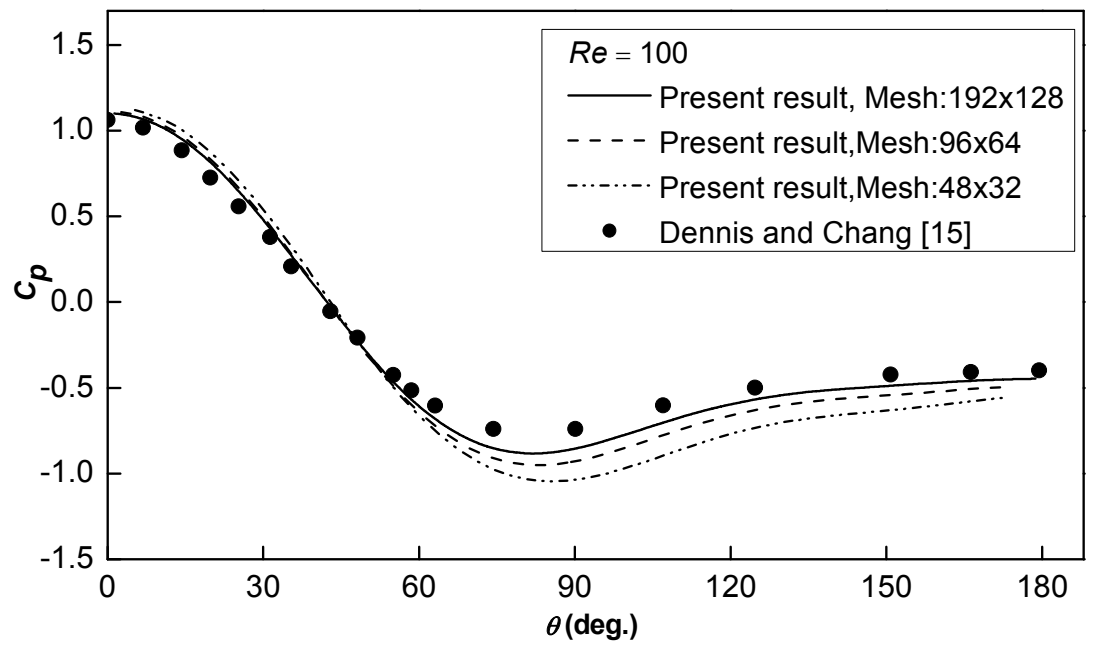

Fig.7d. Pressure coefficients for circular cylinder at (d) $\mathrm{Re}=100$.

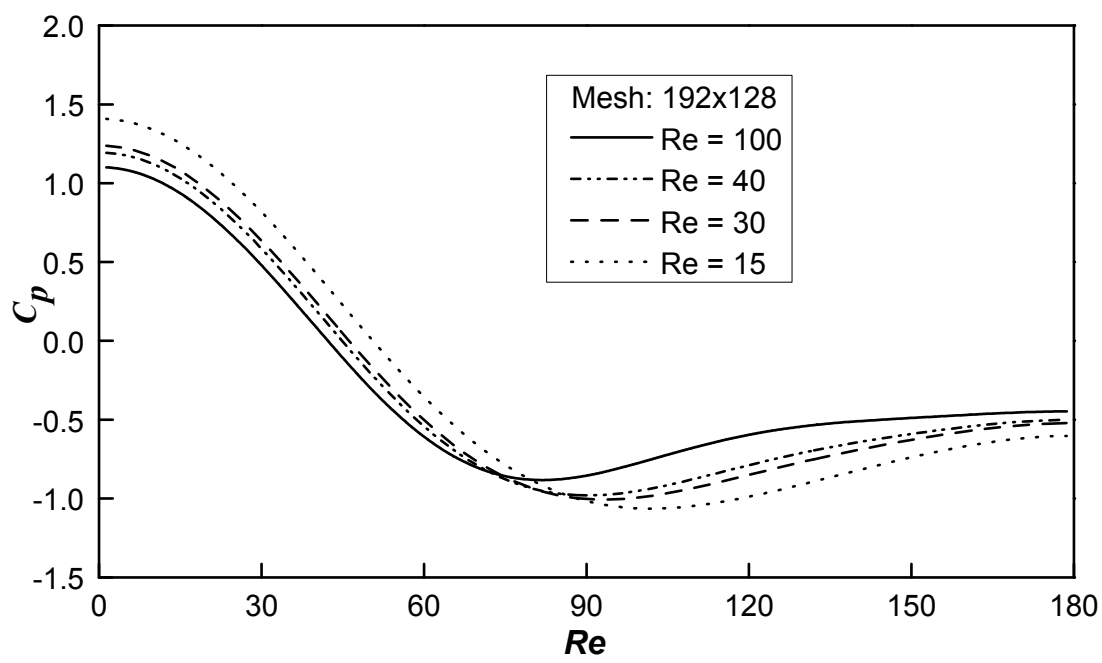

Fig.8. Effect of Reynolds number on pressure coefficient of a circular cylinder.

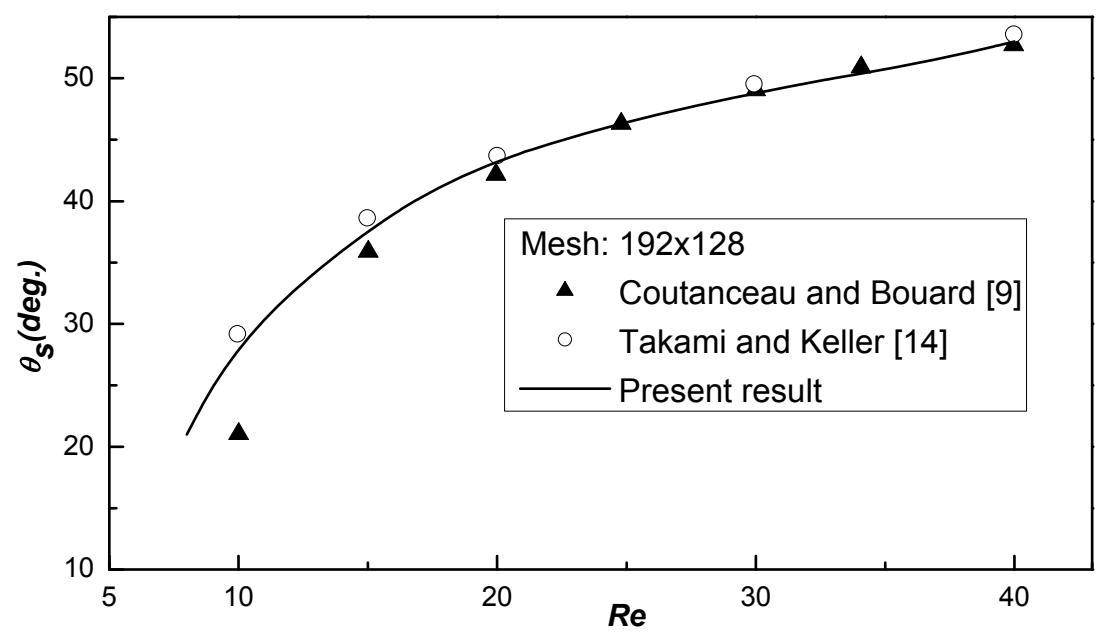

Fig.9. Variation of separation angle with Reynolds number for a circular cylinder. 


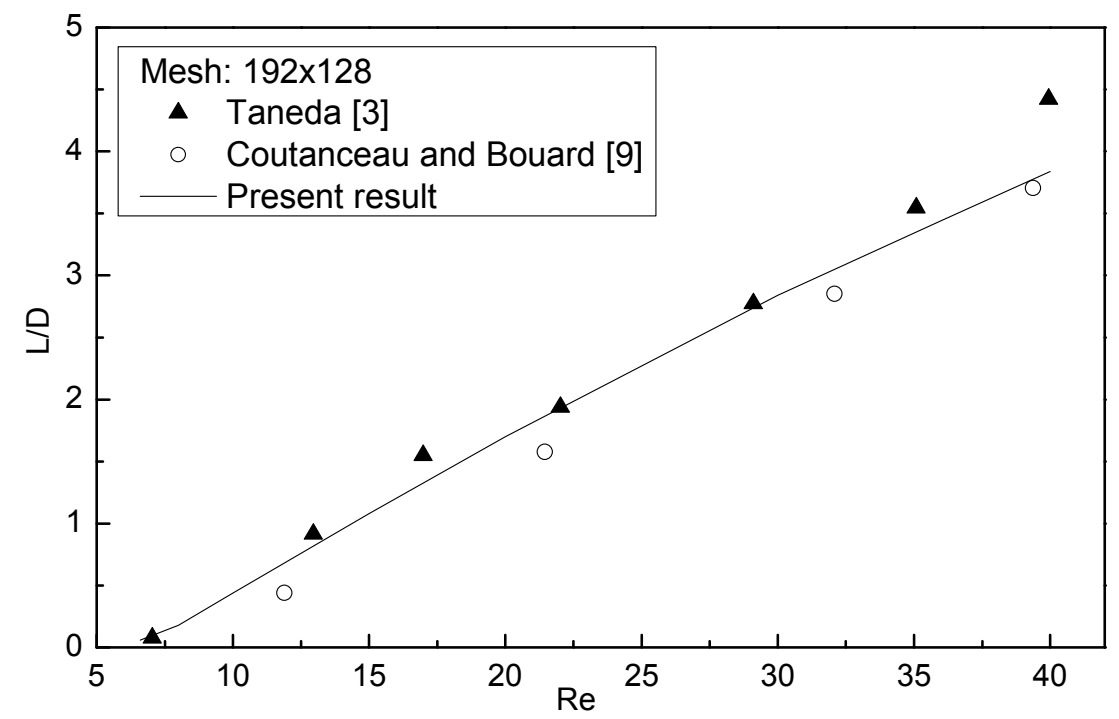

Fig.10. Variation of non-dimensional bubble length with Reynolds number for a circular cylinder.

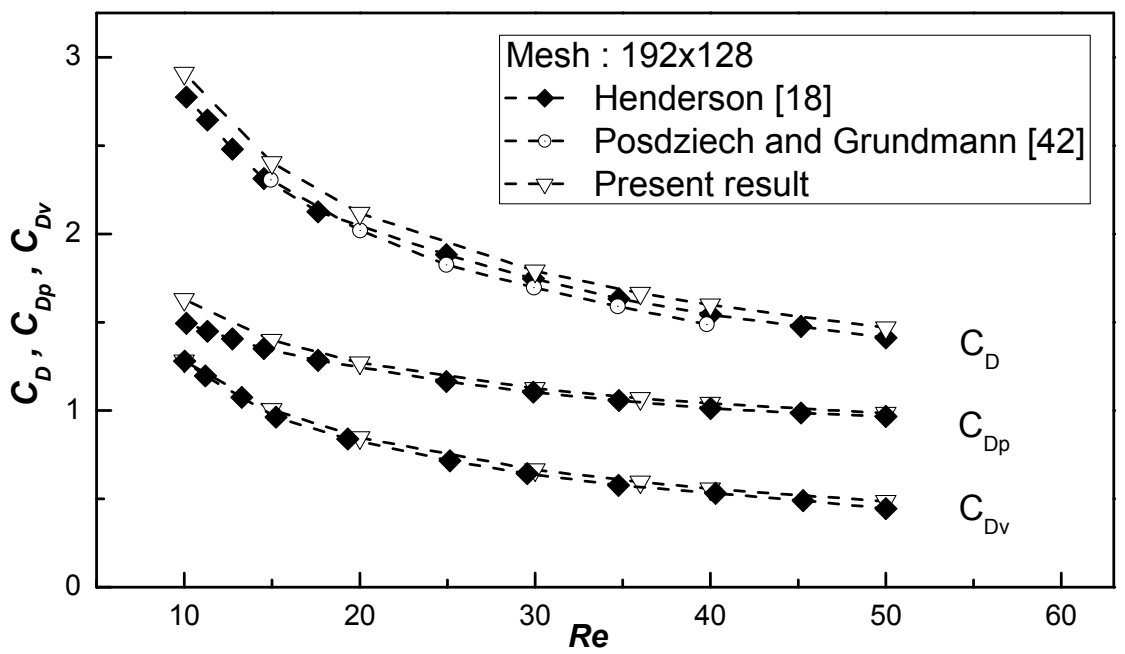

Fig.11. Variation of drag coefficient with Reynolds number for a circular cylinder.

In the present work, functional relations based on the least square fitting has been obtained to predict the variation of the bubble length, separation angle and drag coefficient with the Reynolds number using data in the range $8 \leq \mathrm{Re} \leq 40$ for a steady flow around a circular cylinder. The obtained relations are given below

$$
\begin{array}{ll}
L / r=-0.687+0.115 \mathrm{Re}, & 8 \leq \mathrm{Re} \leq 40, \\
\theta_{S}=78.16-158.02 \mathrm{Re}^{-0.5}, & 8 \leq \mathrm{Re} \leq 40, \\
C_{D}=0.302+8.82 \mathrm{Re}^{-0.5}, & 8 \leq \mathrm{Re} \leq 40 .
\end{array}
$$


For an unbounded flow Sen et al. [21] and Sobey [38] found the following expression for the bubble length

$$
\begin{aligned}
& L / r=-0.847+0.1336 \operatorname{Re}, \quad \operatorname{Re}_{s}<\operatorname{Re} \leq 40, \\
& L / r=-0.506+0.115 \mathrm{Re} .
\end{aligned}
$$

Sen et al. [21], and Wu et al. [20] proposed the following equation for $\theta_{S}$

$$
\begin{array}{ll}
\theta_{S}=77.66-152.65 \mathrm{Re}^{-0.5}, & 10 \leq \mathrm{Re} \leq 40, \\
\theta_{S}=78.5-155.2 \mathrm{Re}^{-0.5}, & 10 \leq \operatorname{Re} \leq 200 .
\end{array}
$$

Smith [39] and Sen et al. [21] proposed $C_{D}-\operatorname{Re}$ variation for a steady flow as follows

$$
\begin{aligned}
& C_{D}=0.5+3.805 \mathrm{Re}^{-0.5}, \\
& C_{D}=0.26+7.89 \mathrm{Re}^{-0.5}, \quad 15 \leq \mathrm{Re} \leq 40 .
\end{aligned}
$$

By comparing with other empirical equations from the literature, very little discrepancy is found in the case of bubble length and separation angle. Although $C_{D}$ is found to vary as $\mathrm{Re}^{-0.5}$, the present $C_{D}-\mathrm{Re}$ relation shows some discrepancy, and agreement with Sen et al. [21] is better than with Smith [39].

The equations obtained for the bubble length, separation angle, drag coefficient are linearly extrapolated to check their validity at the Reynolds number up to 100 . The extrapolated bubble length and separation angle agree quite well with data from the literature as shown in Fig.12 and Fig.13. The extrapolated drag coefficients agree well with Tritton [4] but the agreement with Henderson [18] and Sen [21] is less satisfactory, particularly at lower Reynolds numbers (see Fig.14).

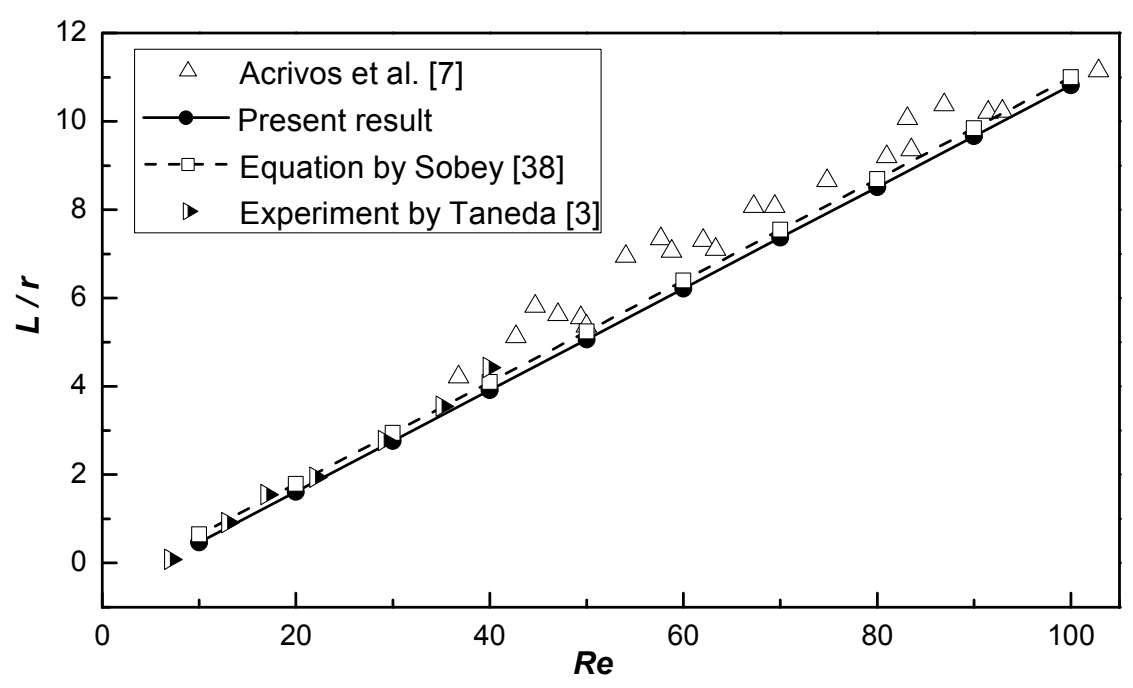

Fig.12. Extrapolated bubble length for circular cylinder. 


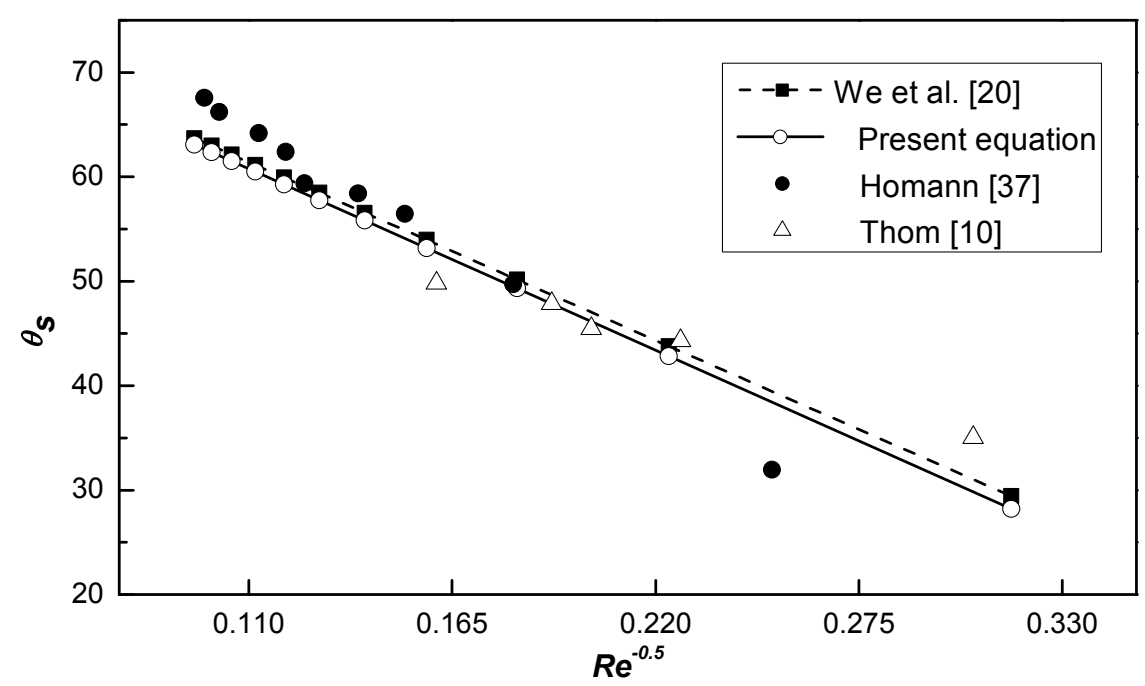

Fig.13. Extrapolated separation angle for circular cylinder.

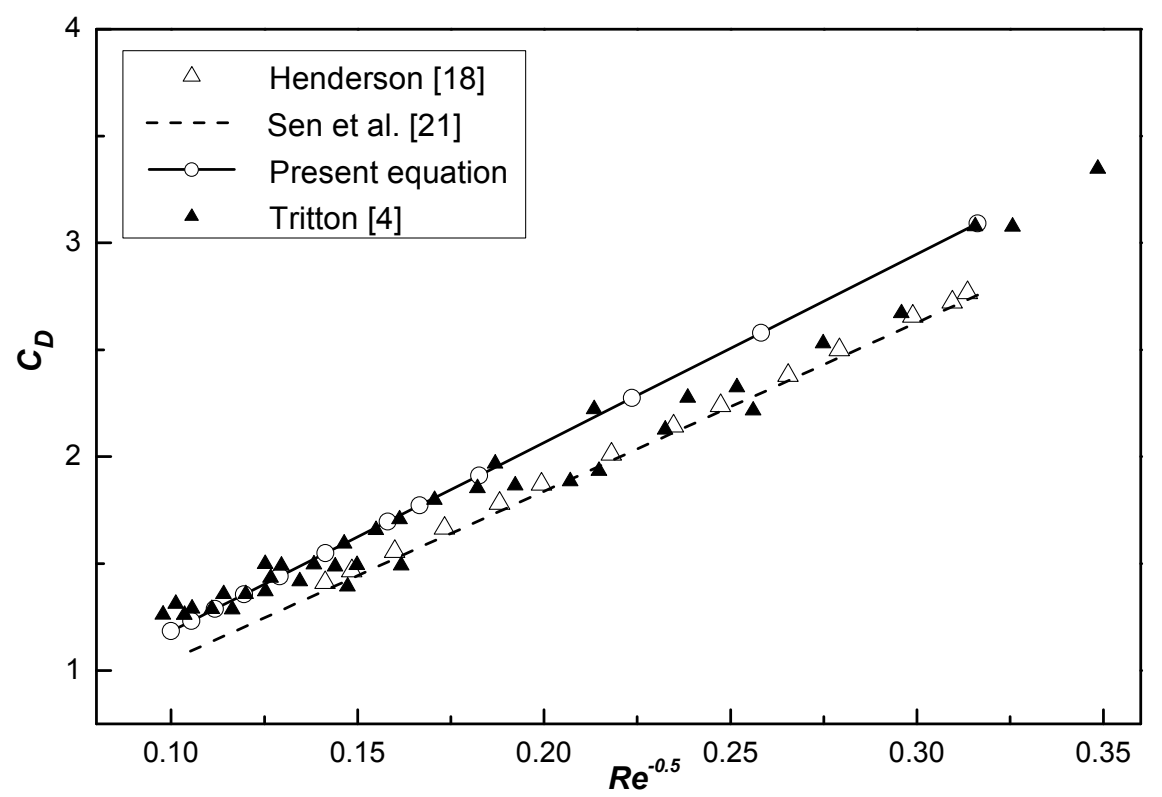

Fig.14. Extrapolated drag coefficients for circular cylinder.

\subsection{Flow past a square cylinder}

Viscous flow past a single square cylinder with unit length and zero incidence is simulated at low Reynolds numbers (Re) with O-type grids of sizes $84 \times 22,168 \times 44,300 \times 88$, and $338 \times 88$. The computational domain details are shown in Fig. 15 and the finest gird is shown in Fig.16. To establish adequacy of the mesh, computations of the bubble length and drag coefficients were carried out at 4 different meshes at $\mathrm{Re}=5$ and 40 . As illustrated by Tab.1, the mesh size of $338 \times 88$ yields grid independent solutions, and hence this grid was used to perform further computations for the square cylinder. 


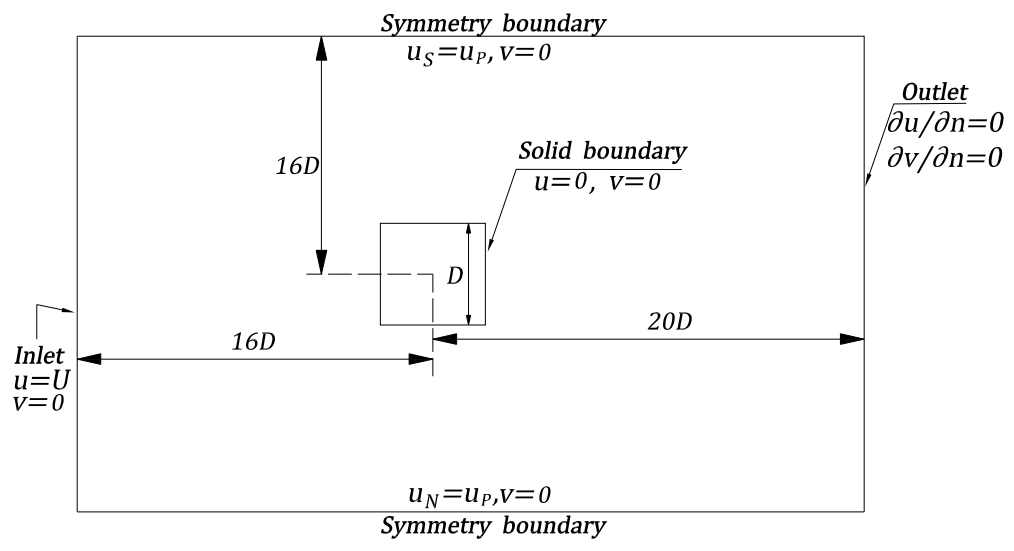

Fig.15. Problem definition and boundary conditions for square cylinder.

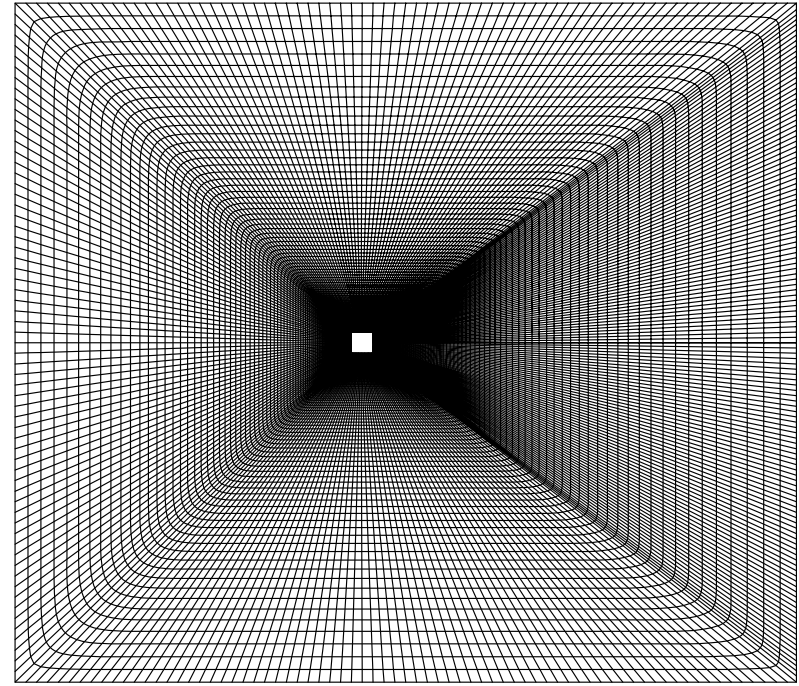

Fig.16. Overview of O-type grid used for a square cylinder.

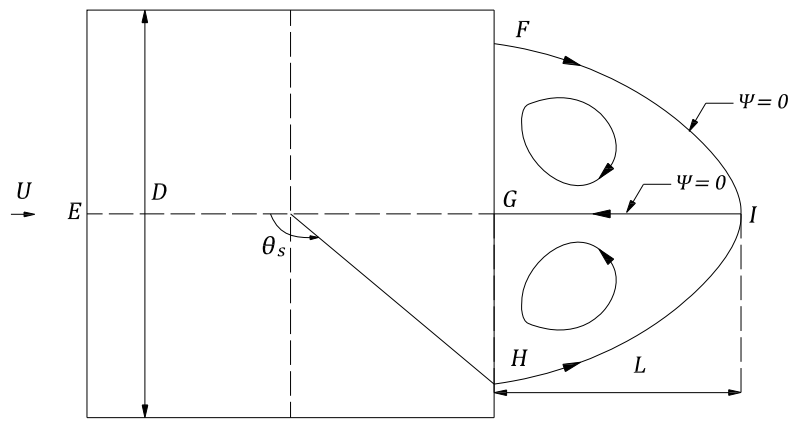

$\mathrm{E}=$ Forward stagnation point

$\mathrm{G}=$ Rear stagnation point

$\mathrm{F}, \mathrm{H}=$ Separation points $\mathrm{I}=$ Wake stagnation point

Fig.17. Schematic representation of the separation bubble cross-section for square

Table 1. Mesh convergence for square cylinder.

\begin{tabular}{cccccc} 
& & \multicolumn{3}{c}{ L/D } & \multicolumn{2}{c}{$\mathrm{C}_{\mathrm{D}}$} \\
\cline { 3 - 6 } Mesh & No. of Cells & 5 & 40 & 5 & 40 \\
\hline & & 0.280 & 2.000 & 4.840 & 1.993 \\
$168 \times 44$ & 7392 & 0.297 & 2.450 & 4.742 & 1.860 \\
$300 \times 88$ & 26400 & 0.299 & 2.673 & 4.680 & 1.786 \\
$336 \times 88$ & 29568 & 0.300 & 2.680 & 4.678 & 1.783 \\
\hline
\end{tabular}

It is evident from Fig. 18 and Fig.19 that the agreement between computed pressure drag coefficients and these of Sen et al. [29] is excellent. The total and viscous drag also shows satisfactory agreement and is only slightly overestimated. The bubble length varies linearly with the Reynolds number and shows an excellent agreement with Paliwal et al. [40] at all Reynolds numbers. The bubble length obtained by the 
present numerical treatment and that by Sen et al. [29] shows 2.4\%-6.6\% discrepancy at Reynolds numbers greater than 32, but agrees very well at lower Reynolds numbers (see Fig.20). The separation angle for the square cylinder (as defined in Fig.17), is plotted against the Reynolds number in Fig.21. It can be seen from Fig.21 that both the present work and Sen et al. [29] predicts the separation angle to reach a limiting value of $135 \mathrm{deg}$ as Reynolds number is increased. A comparison between Fig.11 and Fig.19, and Fig.12 and Fig.20 reveals that, the bubble length $L$, and drag coefficient $C_{D}$ is larger for a cylinder with a square shape compared to that with a circular shape at a given Reynolds number.

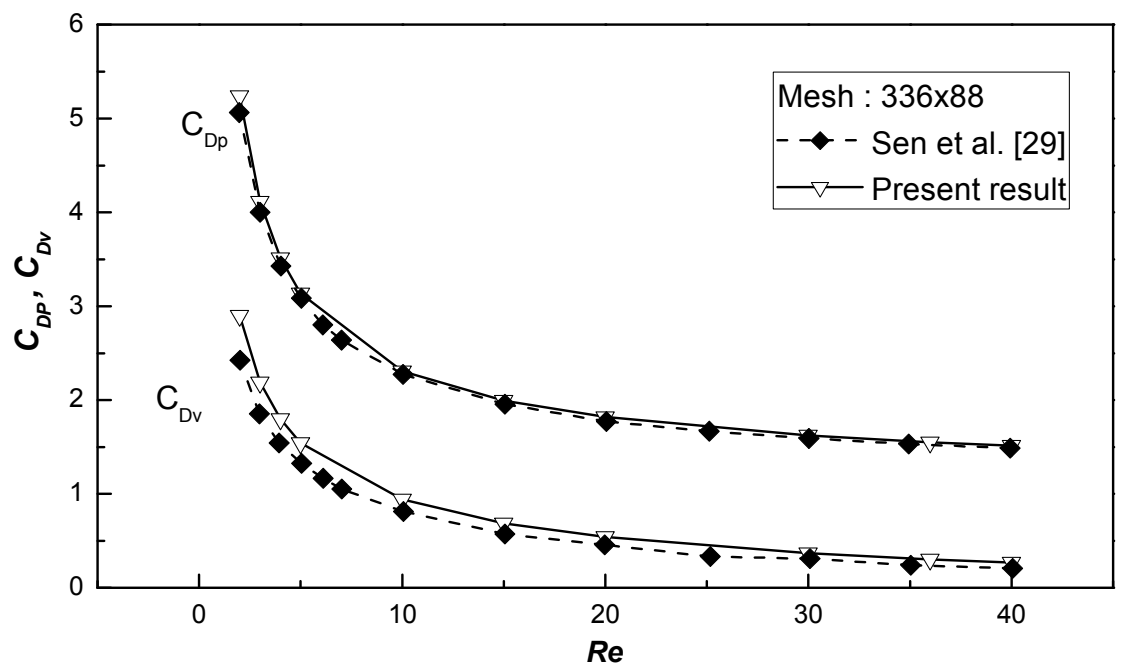

Fig.18. Pressure and viscous drag coefficients vs. Reynolds number for square cylinder.

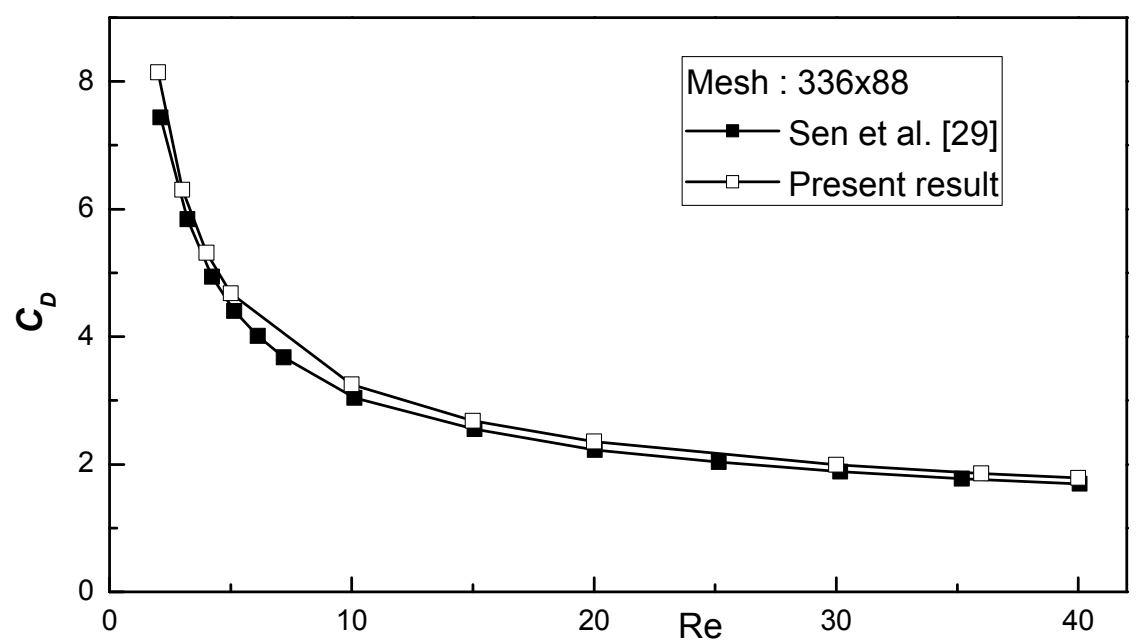

Fig.19. Total Drag coefficient vs. Reynolds number for square cylinder. 


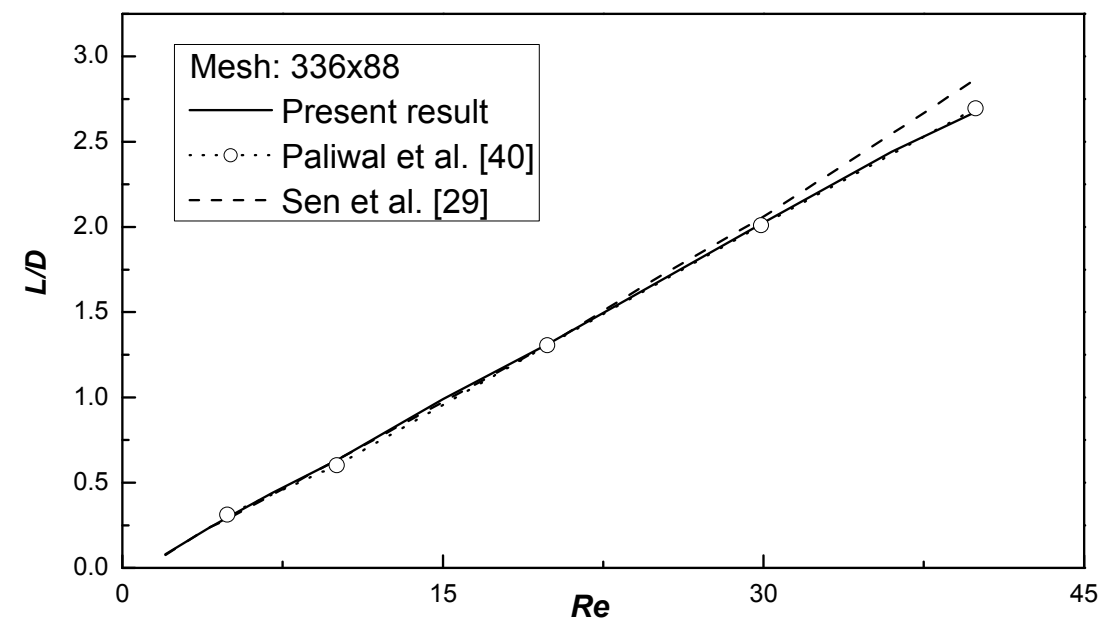

Fig.20. Non-dimensional bubble length vs. Reynolds number for square cylinder.

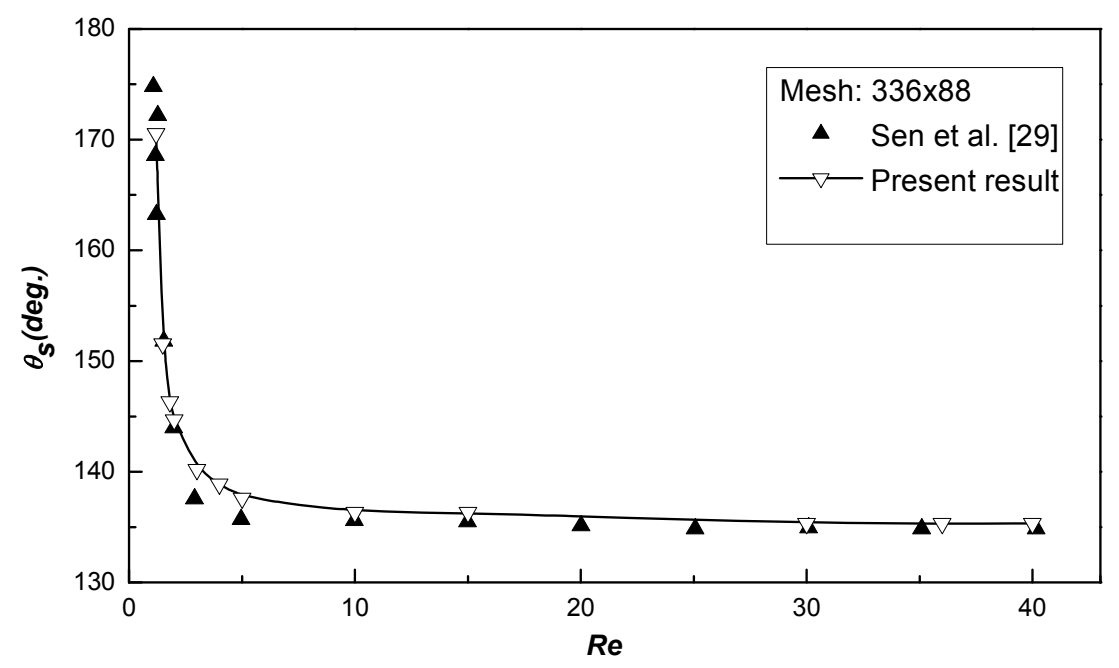

Fig.21. Separation angle vs. Reynolds number for square cylinder.

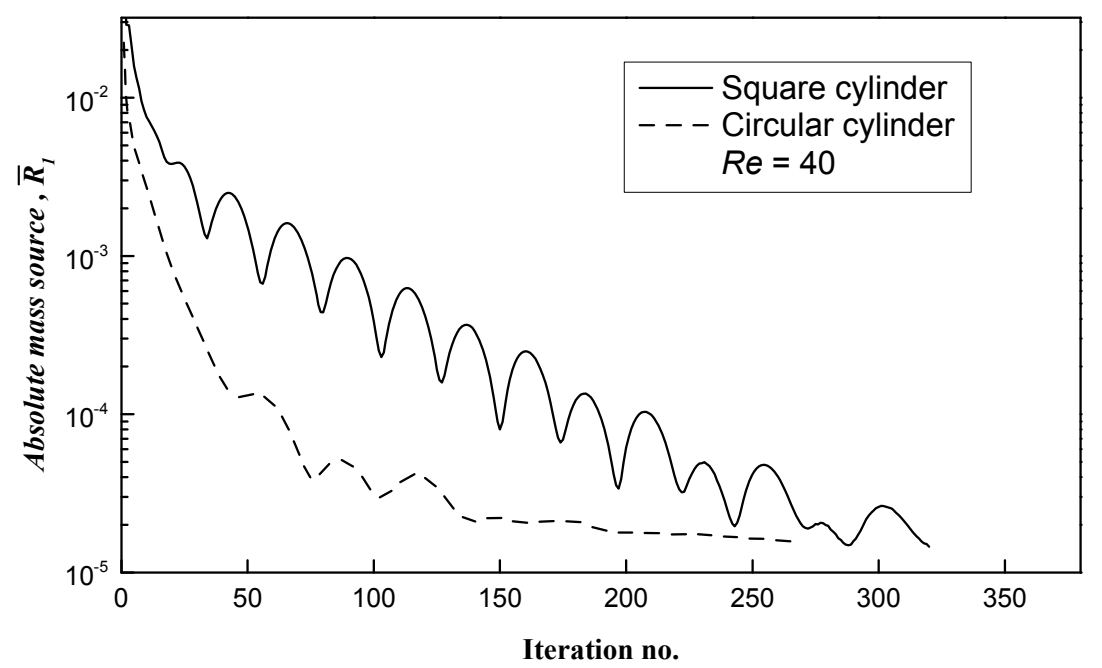

Fig.22. Convergence history. 
Based on the computation between up to 40, empirical relations for of non-dimensional bubble length and drag coefficients are proposed using least square curve fitting. The relation between $L / D, C_{D}$ and Re are given below

$$
\begin{array}{ll}
\frac{L}{D}=-0.047+0.069 \mathrm{Re}, & 5 \leq \operatorname{Re} \leq 40, \\
C_{D}=0.772+11.56 \mathrm{Re}^{-0.666}, & 2 \leq \operatorname{Re} \leq 40 .
\end{array}
$$

The above relations show satisfactory agreement with that of Sen et al. [29] who proposed the following relationships

$$
\begin{array}{ll}
\frac{L}{D}=-0.0783+0.0724 \mathrm{Re}, & 5<\mathrm{Re}<40, \\
C_{D}=0.7496+10.5767 \mathrm{Re}^{-0.66}, & 2<\operatorname{Re}<40 .
\end{array}
$$

In the present numerical methodology, a simplified pressure correction equation was utilized to save computational cost and facilitate the use of the incomplete LU decomposition of Stone [34]. Despite doing so, a satisfactory convergence rate was found by using under -relaxation factors of $\alpha_{u}=\alpha_{v}=0.8, \alpha_{p}=0.2$ for the circular cylinder, and $\alpha_{u}=\alpha_{v}=0.65, \alpha_{p}=0.15$ for the square cylinder. Figure 22 illustrates that despite the simplified pressure correction equation and non-orthogonal grid, the residuals of the continuity equation reduces by a factor of 100 within 60 iterations for circular cylinder and 150 iterations for square cylinder.

\section{Conclusions}

A finite volume discretization method and SIMPLE solution technique with simplified pressure correction equation are used to predict a two-dimensional steady flow past a circular cylinder at blockage of 0.016 and square cylinder at blockage of 0.0625 up to the Reynolds number of 40. Despite neglecting crossderivative terms in the pressure correction equation, a satisfactory convergence rate is found by using underrelaxation factors of $\alpha_{u}=\alpha_{v}=0.8, \alpha_{p}=0.2$ for the circular cylinder and $\alpha_{u}=\alpha_{v}=0.65, \alpha_{p}=0.15$ for the square cylinder.

For the circular cylinder, the onset of flow separation occurs between Reynolds numbers of 6.5-6.6, and the bubble length, drag coefficient, and separation angle obey the following relationships

$$
\begin{array}{ll}
L / r=-0.687+0.115 \mathrm{Re}, & 8 \leq \mathrm{Re} \leq 40, \\
\theta_{S}=78.16-158.02 \mathrm{Re}^{-0.5}, & 8 \leq \mathrm{Re} \leq 40, \\
C_{D}=0.302+8.82 \mathrm{Re}^{-0.5}, & 8 \leq \mathrm{Re} \leq 40 .
\end{array}
$$

The square cylinder has a larger separation bubble and drag coefficients at a given Reynolds number than the circular cylinder, and separation also occurs at lower Reynolds numbers. The separation angle for the square cylinder decreases with an increase in the Reynolds number and tends to a value of 135 deg for $\operatorname{Re}>10$.Moreover, the bubble length and drag coefficient of the square cylinder exposed to a steady viscous flow follows the relationships given below 


$$
\begin{array}{ll}
\frac{L}{D}=-0.047+0.069 \mathrm{Re}, & 5 \leq \mathrm{Re} \leq 40, \\
C_{D}=0.772+11.56 \mathrm{Re}^{-0.666}, & 2 \leq \mathrm{Re} \leq 40 .
\end{array}
$$

By comparing equations given above it can be further concluded that at a given Reynolds number the separation bubble length and drag coefficient are greater in case of a square cylinder compared to a circular one. This implies that a cylinder with a square section is more bluff than the one with a circular section.

\section{Acknowledgement}

The authors are thankful to Dr. Milovan Peric for providing permission to use and modify the code CAFFA. The authors also acknowledge the support provided by the Department of NAME, Bangladesh University of Engineering and Technology, during this research work.

\section{Nomenclature}

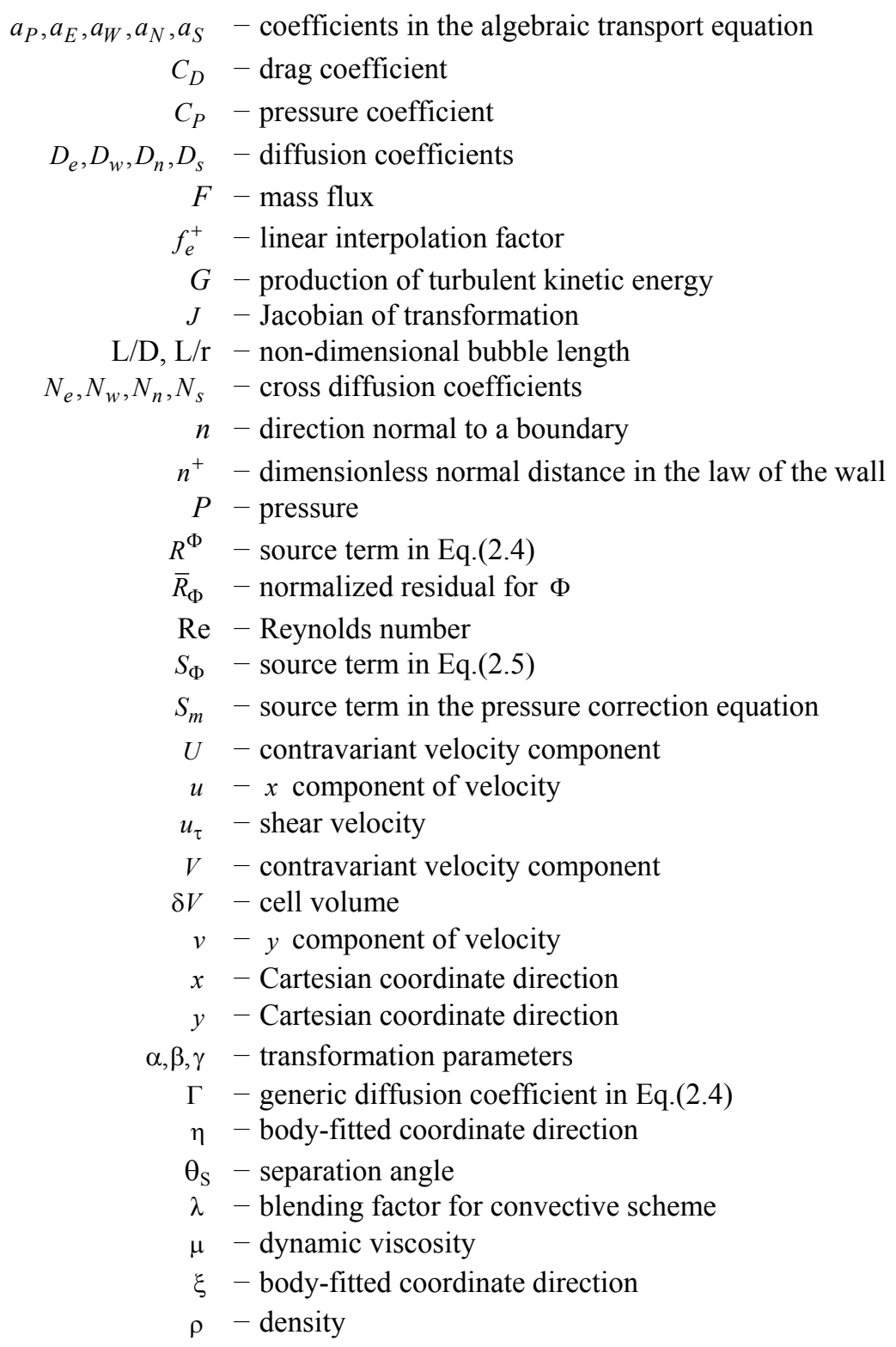




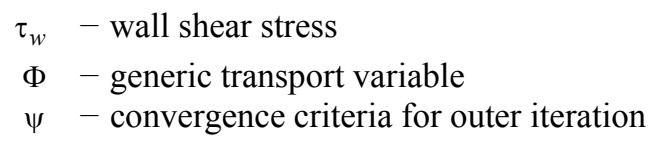

\section{References}

[1] Strouhal V. (1878): Ueber eine besondere Art der Tonerregung. - Annalen der Physik und Chemie, vol.241, pp.216-251.

[2] Nisi H. and Porter A.W. (1923): Philos. Mag., vol.46, pp.754.

[3] Taneda S. (1956): Experimental investigation of the wakes behind cylinders and plates at low Reynolds numbers. - J. Phys. Soc. Japan, vol.11, pp.302-307.

[4] Tritton D.J. (1959): Experiments on the flow past a circular cylinder at low Reynolds numbers. - J. Fluid Mech. vol.6, pp.547-567.

[5] Grove A.S., Shair F.H., Peterson E.E. and Acrivos A. (1964): An experimental investigation of the steady separated flow past a circular cylinder. - J. Fluid Mech. vol.19, pp.60-80.

[6] Acrivos A., Snowden D.D., Grove A.S. and Peterson E.E. (1965): The steady separated flow pasta circular cylinder at large Reynolds numbers. - J. Fluid Mech., vol.21, pp.737-760.

[7] Acrivos A., Leal L.G., Snowden D.D. and Pan F. (1968): Further experiments on steady separated flows past bluff objects. - J. Fluid Mech., vol.34, pp.25-48.

[8] Nishioka M. and Sato H. (1974): Measurements of velocity distributions in the wake of a circular cylinder at low Reynolds numbers. - J. Fluid Mech., vol.65, pp.97-112.

[9] Coutanceau M. and Bouard R. (1977): Experimental determination of the main features of the viscous flow in the wake of a circular cylinder in uniform translation. Part 1. Steady flow. - J. Fluid Mech., vol.79, pp.231-256.

[10] Thom A. (1933): The flow past circular cylinders at low speeds. - Proc. R. Soc. Lond. A, vol.141, pp.651-669.

[11] Kawaguti M. (1953): Numerical solution of the Navier-Stokes equations for the flow around a circular cylinder at Reynolds number 40. - J. Phys. Soc. Jpn, vol.8, pp.747-757.

[12] Apelt C.J. (1961): The steady flow of a viscous fluid past a circular cylinder at Reynolds numbers40 and $44 .-$ Aeronaut. Res. Counc. Lond. R \& M, vol.3175, pp.1-28.

[13] Kawaguti M. and Jain P. (1966): Numerical study of a viscous fluid flow past a circular cylinder. - J. Phys. Soc. Jpn, vol.21, pp.2055-2062.

[14] Takami H. and Keller H.B. (1969): Steady two-dimensional viscous flow of an incompressible fluid past a circular cylinder. - Phys. Fluids Suppl., vol.12, pp.51-56.

[15] Dennis S.C.R. and Chang G.Z. (1970): Numerical solutions for steady flow past a circular cylinder at Reynolds numbers up to 100. - J. Fluid Mech., vol.42, pp.471-489.

[16] Fornberg B. (1980): A numerical study of steady viscous flow past a circular cylinder. - J. Fluid Mech., vol.98, pp.819-855.

[17] Fornberg B. (1985): Steady viscous flow past a circular cylinder up to Reynolds number 600. - J. Comput. Phys., vol.61, pp.297-320.

[18] Henderson R.D. (1995): Details of the drag curve near the onset of vortex shedding. - Phys. Fluids, vol.7, pp.2102-2104.

[19] Chen J.H. (2000): Laminar separation of flow past a circular cylinder between two parallel plates. - Proc. Natl Sci. Counc. ROC A, vol.24, pp.341-351.

[20] Wu M.H., Wen C.Y., Yen R.H., Weng M.C. and Wang A.B. (2004): Experimental and numerical study of the separation angle for flow around a circular cylinder at low Reynolds number. - J. Fluid Mech., vol.515, pp.233-260.

[21] Sen S., Mittal S. and Biswas G. (2009): Steady separated flow past a circular cylinder at low Reynolds numbers. - J. Fluid Mech., vol.620, pp.89-119. 
[22] Wei D.J., Yoon H.S. and Jung J.H. (2016): Characteristics of aerodynamic forces exerted on a twisted cylinder at a low Reynolds number of 100. - Comput. Fluids, vol.136, pp.456-466.

[23] Okajima A. (1982): Strouhal numbers of rectangular cylinders. - J. Fluid Mech., vol.123, pp.379-398.

[24] Okajima A., Nagashisa T. and Rokugoh A. (1990): A numerical analysis of flow around rectangular cylinders. - JSME Int. Series II, vol.33, pp.702-717.

[25] Mukhopadhaya A., Biswas, G. and Sundararajan T. (1992): Numerical investigation of confined wakes behind a square cylinder in a channel. - Int. J. Numer. Methods Fluids, vol.14, pp.1437-1484.

[26] Sohankar A., Norberg C. and Davidson L. (1998): Low-Reynolds-number flow around a square cylinder at incidence: study of blockage, onset of vortex shedding and outlet boundary condition. - Int. J. Numer. Methods Fluids, vol.26. pp.39-56.

[27] Breuer M., Bernsdorf J., Zeiser T. and Durst F. (2000): Accurate computations of the laminar flow past a square cylinder based on two different methods: lattice-Boltzmann and finite-volume. - Int. J. Heat Fluid Flow, vol.21, pp.186-196.

[28] Gupta A.K., Sharma A., Chhabra R.P. and Eswaran V. (2003): Two-dimensional steady flow of a power-law fluid past a square cylinder in a plane channel: momentum and heat-transfer characteristics. - Ind. Eng. Chem. Res., vol.42, pp.5674-5686.

[29] Sen S., Mittal S. and Biswas, G. (2010): Flow past a square cylinder at low Reynolds numbers. - Int. J. Numer. Methods Fluids, vol.67. pp.1160-1174.

[30] Mahir N. (2017): Three dimensional heat transfer from a square cylinder at low Reynolds numbers. - Int. J. Thermal Sciences, vol.119, pp.37-50.

[31] Patanker S.V. (1980): Numerical Heat Transfer and Fluid Flow. - New York: McGraw-Hill.

[32] Demirdzic I. and Peric M. (1990): Finite volume method for prediction of fluid flow in arbitrary shaped domains with moving boundary. - Int. J. Numer. Methods Fluids, vol.10, pp.771-790.

[33] Patanker S.V. and Spalding D.B. (1972): A calculation procedure for heat, mass and momentum transfer in three-dimensional parabolic flows. - Int. J. Heat Mass Transfer, vol.15, pp.1787-1806.

[34] Stone H.L. (1968): Iterative solution of implicit approximations of multidimensional partial differential equations. - SIAM. J. Numerical Analysis, vol.5, pp.530-558.

[35] Rhie C.M. (1981): A Numerical Study of the Flow Past an Isolated Airfoil with Separation. - PhD Thesis, Dept. of Mechanical and Industrial Engineering. University of Illinois at Urbana-Champaign.

[36] Peric M. (1990): Analysis of pressure-velocity coupling on non-orthogonal grids. - Numer. Heat Transfer, Part B, vol.17, pp.63-82.

[37] Homann F. (1936): Einfluss grosser zahigkeit bei stromung um zylinder. - Forsch. Ing. Wes., vol.7, pp.1-10.

[38] Sobey I.J. (2000): Introduction to Interactive Boundary Layer Theory. - Oxford University Press.

[39] Smith F.T. (1981): Comparisons and comments concerning recent calculations for flow past a circular cylinder. - J. Fluid Mech., vol.113, pp.407-410.

[40] Paliwal B., Sharma A., Chhabra R.P. and Eswaran V. (2003): Power law fluid flow past a square cylinder: momentum and heat transfer characteristics. - Chem. Eng. Sci., vol.58, pp.5315-5329.

[41] Hamielec A.E. and Raal J.D. (1969): Numerical studies of viscous flow around circular cylinders. - Phys. Fluids., vol.12, pp.11-17.

[42] Posdziech O. and Grundmann R. (2007): A systematic approach to the numerical calculation of fundamental quantities of the two-dimensional flow over a circular cylinder. - J. Fluids Struct., vol.23, pp.479-499.

Received: June 21, 2019

Revised: March 26, 2020 